\title{
ON THE NUMERICAL MODELING OF DEFORMATIONS OF PRESSURIZED MARTENSITIC THIN FILMS*
}

\author{
PAvel BĚlík ${ }^{1}$, Timothy Brule ${ }^{1}$ And Mitchell Luskin ${ }^{1}$
}

\begin{abstract}
We propose, analyze, and compare several numerical methods for the computation of the deformation of a pressurized martensitic thin film. Numerical results have been obtained for the hysteresis of the deformation as the film transforms reversibly from austenite to martensite.
\end{abstract}

Mathematics Subject Classification. 49J45, 65N15, 65N30, 74B20, 74G65, 74K35, 74S05.

Received: April 17, 2000. Revised: November 24, 2000.

\section{INTRODUCTION}

Active martensitic thin films are increasingly being utilized in new and proposed technologies $[7,32]$. The development of techniques for the growth of single crystal martensitic thin films [23] offers the promise of even larger work output per cycle [32]. Recently, Bhattacharya and James have derived a thin film model for martensitic thin films [7] from the geometrically nonlinear bulk theory of martensite $[4,5,15,41]$. We have utilized the Bhattacharya-James model to develop several finite element models for the deformation of active martensitic thin films.

If the film is deposited on a substrate in the austenitic phase and it is released in a square region, then it can pop up and form a "tent" in the martensitic phase (Fig. 1) if the transformation strain satisfies compatibility conditions (6.1)-(6.3) [7]. Such a "tent" deformation has been proposed for use in a temperature activated micro-valve (Fig. 2).

We propose and utilize an energy density for a cubic to tetragonal transformation that satisfies the conditions (6.1)-(6.3) for the "tent" deformation. In this paper, several finite element approximations for the deformation are tested and compared. We computed the deformation of a pressurized film by continuation in the temperature as the film is cyclically cooled and heated through the transformation temperature and obtained nearly rectangular hysteresis in the deformation (Fig. 5).

We have found in our model that the corners where the film is attached to the substrate provide a nucleation mechanism for the phase transformation from martensite to austenite. The discontinuous Morley element gave unstable results under mesh refinement for the transformation temperature. We believe this is because the

\footnotetext{
Keywords and phrases. Thin film, finite element, martensitic transformation, active materials.

* This work was supported in part by NSF DMS 95-05077 and 00-74043, by AFOSR F49620-98-1-0433, by ARO DAAG55-

98-1-0335, by the Institute for Mathematics and Its Applications, and by the Minnesota Supercomputer Institute.

1 School of Mathematics, University of Minnesota, 206 Church Street SE, Minneapolis, MN 55455 USA.

e-mail: belik@math.umn.edu; e-mail: brule@math.umn.edu; e-mail: luskin@math.umn.edu
} 
Morley element does not accurately model the deformation in the corner. More stable results were obtained with the reduced Hsieh-Clough-Tocher element and the Zienkiewicz element, both of which are continuous elements.

An analysis of the convergence of the finite element approximation of the thin film model for martensitic crystals is given in this paper. We have developed a stability theory and analysis of the finite element approximation for bulk martensitic crystals in $[8,11,35-37,40,41]$. The stability theory was also used to analyze the microstructure in ferromagnetic crystals in [42]. Related results on the numerical analysis of nonconvex variational problems can be found, for example, in [12-14,16, 22, 29,33,38,44,46].

In Section 2, the Bhattacharya-James thin film model is given, and in Section 3, the finite element spaces that we use to approximate the thin film model are described. In Section 4, we describe the optimization and continuation methods that we use to compute minima for the energy as the temperature or pressure are varied. In Section 5, rigorous results for the convergence of the finite element approximation are given, and in Section 6 , the results of our numerical experiments are given and intrepreted.

\section{THE THIN FILM MODEL}

\subsection{General settings}

We assume that $\Omega \subset \mathbb{R}^{2}$ is a bounded domain with a Lipschitz continuous boundary, $\partial \Omega$. We consider functions whose domain is $\Omega$ and range is $\mathbb{R}^{3}$. The gradient of a typical such function $y$ will be a function whose domain is $\Omega$ and range is $\mathbb{R}^{3 \times 2}$, the set of all real $3 \times 2$ matrices, and will be denoted by $\nabla y$. The columns of $\nabla y$ will be denoted by $y_{, i}, i=1,2$. For a matrix $A \in \mathbb{R}^{3 \times 3}$, we also write $A=\left(A_{12} \mid A_{3}\right)$ where $A_{i}, i=1,2,3$, represent the columns of $A$, and $A_{12}$ represents the $3 \times 2$ submatrix of $A$ formed by the columns $A_{1}$ and $A_{2}$. We define $y_{i, \alpha}=\partial y_{i} / \partial x_{\alpha}$ for $i=1,2,3$ and $\alpha=1,2, y_{i, \alpha \beta}=\partial^{2} y_{i} / \partial x_{\alpha} \partial x_{\beta}$ for $i=1,2,3$ and $\alpha, \beta=1,2$, and denote by $\nabla^{2} y$ the $3 \times 2 \times 2$ tensor of all second order partial derivatives of $y$. We equip all matrix spaces with the usual component-wise inner products and the corresponding norms. The elastic energy density is denoted by the function $\phi(F, \theta): \mathbb{R}^{3 \times 3} \times \mathbb{R} \rightarrow \mathbb{R}$. Finally, the temperature will be denoted by $\theta$ and the applied hydrostatic pressure by $P$.

Bhattacharya and James [7] and James and Rizzoni [31] have shown that deformations $u \in H^{2}\left(\Omega_{h} ; \mathbb{R}^{3}\right)$ minimizing the bulk energy of a pressurized film of thickness $h$ with reference configuration

$$
\Omega_{h} \equiv \Omega \times(-h / 2, h / 2)
$$

are asymptotically of the form (see $[7,31]$ for a precise description of the results)

$$
u\left(x_{1}, x_{2}, x_{3}\right)=y\left(x_{1}, x_{2}\right)+b\left(x_{1}, x_{2}\right) x_{3}+\mathrm{o}\left(x_{3}^{2}\right) \quad \text { for }\left(x_{1}, x_{2}, x_{3}\right) \in \Omega_{h},
$$

where $(y, b) \in H^{2}\left(\Omega ; \mathbb{R}^{3}\right) \times H^{1}\left(\Omega ; \mathbb{R}^{3}\right)$ minimizes

$$
\mathcal{E}_{\kappa, \theta, P}(y, b)=\int_{\Omega}\left\{\kappa\left(\left|\nabla^{2} y\right|^{2}+2|\nabla b|^{2}\right)+\phi(\nabla y \mid b, \theta)-P y_{3}\left(y_{1,1} y_{2,2}-y_{1,2} y_{2,1}\right)\right\} \mathrm{d} x
$$

among all pairs $(y, b) \in H^{2}\left(\Omega ; \mathbb{R}^{3}\right) \times H^{1}\left(\Omega ; \mathbb{R}^{3}\right)$ satisfying prescribed Dirichlet boundary conditions

$$
(y(x), b(x))=(\hat{y}(x), \hat{b}(x)) \quad \text { for } x \in \Gamma,
$$

where $\Gamma \subset \partial \Omega$ is a relatively open set of positive one-dimensional Hausdorff measure and $(\hat{y}, \hat{b}) \in H^{2}\left(\Omega ; \mathbb{R}^{3}\right) \times$ $H^{1}\left(\Omega ; \mathbb{R}^{3}\right)$. Let us define the space of admissible deformations $\mathcal{A}$ by

$$
\mathcal{A}=\left\{(y, b) \in H^{2}\left(\Omega ; \mathbb{R}^{3}\right) \times H^{1}\left(\Omega ; \mathbb{R}^{3}\right):(y, b)=(\hat{y}, \hat{b}) \text { on } \Gamma\right\} .
$$


We note that $\mathcal{A}=\mathcal{M} \times \mathcal{N}$ where

$$
\mathcal{M}=\left\{y \in H^{2}\left(\Omega ; \mathbb{R}^{3}\right): y=\hat{y} \text { on } \Gamma\right\} \quad \text { and } \quad \mathcal{N}=\left\{b \in H^{1}\left(\Omega ; \mathbb{R}^{3}\right): b=\hat{b} \text { on } \Gamma\right\} .
$$

The requirement that the film not penetrate itself [30] is not included in the definition of the space of admissible deformations, $\mathcal{A}$. However, this requirement has been satisfied by all of the numerical solutions that we have obtained with this model with the admissible space given by (2.3).

The deformation of the film, whose reference (undistorted) configuration is given by the domain $\Omega$, is described by $y$, while the deformation of the cross-section can be thought of as being described by $b$. The first term of the energy functional is the strain-gradient term modeling the interfacial energy of the thin film and is the limit of the corresponding term for a three-dimensional thin film as the thickness of the film tends to zero. Here $\kappa$ is a small material constant, called the strain-gradient coefficient.

The second term of the energy functional, $\int_{\Omega} \phi(\nabla y \mid b, \theta) \mathrm{d} x$, models the elastic energy of the film at the temperature $\theta$, and it is the limit of the corresponding three-dimensional elastic energy. The third term of the energy functional models the effect of applying uniform hydrostatic pressure $P \geq 0$ under the surface of the film. This term has the form $P \times V$ (pressure $\times$ volume) where $V$ denotes the volume under the distorted film [31].

\subsection{Boundary conditions}

The "tent" deformation can be obtained from a film that is attached to a film in the austenitic phase on its boundary and satisfies certain compatibility conditions [7]. The boundary conditions are

$$
\left.\begin{array}{l}
\left(y_{1}\left(x_{1}, x_{2}\right), y_{2}\left(x_{1}, x_{2}\right), y_{3}\left(x_{1}, x_{2}\right)\right)=\left(x_{1}, x_{2}, 0\right) \\
\left(b_{1}\left(x_{1}, x_{2}\right), b_{2}\left(x_{1}, x_{2}\right), b_{3}\left(x_{1}, x_{2}\right)\right)=(0,0,1)
\end{array}\right\} \text { for }\left(x_{1}, x_{2}\right) \in \partial \Omega
$$

\subsection{Energy density $\phi$}

The requirement that deformations not be penetrating [30] implies that the energy density $\phi$ need only be defined on $\mathbb{R}_{+}^{3 \times 3} \times \mathbb{R}$ where $\mathbb{R}_{+}^{3 \times 3}$ denotes the set of matrices $F \in \mathbb{R}^{3 \times 3}$ such that $\operatorname{det} F>0$. However, we shall assume for simplicity that $\phi$ is defined on all of $\mathbb{R}^{3 \times 3} \times \mathbb{R}$, and we note that the energy density that we propose in (6.6) and use in our numerical computations is defined on all of $\mathbb{R}^{3 \times 3} \times \mathbb{R}$.

We assume that $\phi$ is rotationally invariant, or frame indifferent, that is, for any $\theta \in \mathbb{R}$,

$$
\phi(R F, \theta)=\phi(F, \theta) \quad \text { for all } F \in \mathbb{R}^{3 \times 3} \text { and } R \in \mathrm{SO}(3),
$$

where $\mathrm{SO}(3)$ is the group of rotations, and also that it inherits the symmetry of the more symmetric, high temperature phase of the crystal, so that, for any $\theta \in \mathbb{R}$,

$$
\phi\left(F R_{i}, \theta\right)=\phi(F, \theta) \quad \text { for all } F \in \mathbb{R}^{3 \times 3} \text { and } R_{i} \in \mathcal{G},
$$

where $\mathcal{G} \subset \mathrm{SO}(3)$ is the symmetry group of the austenite.

We assume that near the transformation temperature the energy density has a local minimum at the identity deformation gradient $I$ corresponding to the austenitic phase, and also at the set of variants

$$
\left\{U_{1}, \ldots, U_{M}\right\}=\left\{R_{i} U_{1} R_{i}^{T}: R_{i} \in \mathcal{G}\right\},
$$

where the $U_{i}$ are deformation gradients for an unstressed crystal in the martensitic phase. From the frame indifference (2.6) and from the material symmetry (2.7), it follows that $\phi$ has local minima on the set $\mathrm{SO}(3)$ and on the union of the energy wells

$$
\mathcal{U}=\mathcal{U}_{1} \cup \cdots \cup \mathcal{U}_{M},
$$


where $\mathcal{U}_{i}=\mathrm{SO}(3) U_{i}$. We note that $\phi$ is constant on $\mathcal{U}$ by the frame indifference (2.6) and the material symmetry (2.7). We model the phase transformation by assuming that the set of global minimizers of $\phi$ is exactly the set of rotations $\mathrm{SO}(3)$ for temperatures above the transformation temperature, the set $\mathcal{U}$ for temperatures below the transformation temperature, and the union $\mathcal{U} \cup \mathrm{SO}(3)$ at the transformation temperature.

Since $\mathcal{U}_{i}=\mathrm{SO}(3) U_{i}$, it follows from $(2.8)$ that $\operatorname{det} U$ is constant for all $U \in \mathcal{U}$. We assume that the minimizing deformations are orientation preserving, that is,

$$
\operatorname{det} U=\operatorname{det} U_{1}>0 \quad \text { for all } U \in \mathcal{U} \text {. }
$$

We also assume that the free energy density $\phi$ is continuous, and that $\phi$ satisfies certain growth conditions that will be described in detail in Section 5 .

We focus on the cubic to tetragonal transformation in Section 6.3, and we give there the energy density $\phi$ that we choose for our numerical experiments.

\section{Finite Elements}

In our computations, we have experimented with various conforming as well as nonconforming finite elements. To construct a conforming finite element space for the deformation $y$, one needs to ensure that the basis functions are of class $\mathcal{C}^{1}(\bar{\Omega})[17]$. This results in more complicated spaces than if nonconforming finite element spaces are used. We implemented two conforming elements and two nonconforming elements for the deformation vector $y$, together with a corresponding conforming $\left(\mathcal{C}^{0}(\bar{\Omega})\right)$ or nonconforming $\mathcal{P}_{1}$ element for the vector $b$.

As the first conforming finite element, we implemented the Argyris triangle $[2,9,17]$ for $y$ and the conforming $\mathcal{P}_{1}$ element $[9,17]$ for $b$. The large number of degrees of freedom (21) for the Argyris triangle made this element less efficient for the resolution that we require for our problem than the reduced Hsieh-Clough-Tocher (HCT) finite element described in the following paragraph.

As the second conforming finite element, we implemented the reduced Hsieh-Clough-Tocher (HCT) finite element $[6,9,17,18]$ for $y$, together with the conforming $\mathcal{P}_{1}$ element for $b$. The reduced HCT finite element space consists of functions that are of class $\mathcal{C}^{1}(\bar{\Omega})$ and are such that, when restricted to an element, they are cubic polynomials on each of three triangular subdomains, patched together in such a way so as to form a $\mathcal{C}^{1}(\Omega)$ function. This element has 9 local degrees of freedom.

We implemented the nonconforming, but $\mathcal{C}^{0}(\bar{\Omega})$, Zienkiewicz element $[17,34]$ for $y$, but in this case we used again the conforming $\mathcal{P}_{1}$ element for $b$. The main reason is that the reduced HCT triangle and the Zienkiewicz triangle have exactly the same degrees of freedom (the values and the first partial derivatives at the nodes of the triangle) and therefore the result of one of the two methods can be used as an initial guess (or preconditioner) for the other one - as long as the elements used for $b$ are the same. The Zienkiewicz finite element space consists of functions $v$ that are continuous on $\bar{\Omega}$ and when restricted to an element $K$ of the triangulation they belong to the space $\mathcal{P}_{3}$, the space of cubic polynomials, satisfying the constraint

$$
v\left(\frac{A_{1}+A_{2}+A_{3}}{3}\right)=\frac{1}{3} \sum_{i=1}^{3} v\left(A_{i}\right)+\frac{1}{18} \sum_{\substack{1 \leq j, k \leq 3 \\ j \neq k}} D v_{j, k}
$$

where the $A_{i}$ denote the vertices of $K$ and $D v_{j, k}=\nabla v\left(A_{j}\right) \cdot\left(A_{k}-A_{j}\right)$.

We also implemented the simplest nonconforming element one can use for fourth-order problems, the Morley element $[9,17,43,49]$, which is not $\mathcal{C}^{0}(\Omega)$. The Morley finite element space consists of functions that are elementwise quadratic polynomials and that are moreover continuous at the midpoints of the edges of the triangulation. For this reason, we used the nonconforming $\mathcal{P}_{1}$ element $[9,17]$ for $b$ corresponding to the finite element space consisting of element-wise linear functions which are also continuous at the midpoints of the triangulation edges. 


\section{NUMERICAL MiNimizATION METHOD}

In this section, we describe the gradient minimization method we use to approximate the minimizer of the total energy (2.1). It is based on variants of conjugate gradient methods used in [19-21] with a simplification that increases the efficiency.

Let $\mathcal{M}_{h}$ and $\mathcal{N}_{h}$ denote the finite element spaces approximating the spaces $\mathcal{M}$ and $\mathcal{N}$, respectively, defined in (2.4). In general, the boundary conditions (2.2) for $\mathcal{M}$ and $\mathcal{N}$ will have to be approximated in the definition of $\mathcal{M}_{h}$ and $\mathcal{N}_{h}$. Define $\mathcal{M}_{h, 0}$ and $\mathcal{N}_{h, 0}$ to be the same spaces as $\mathcal{M}_{h}$ and $\mathcal{N}_{h}$, respectively, but with the Dirichlet boundary conditions on $\Gamma$ replaced by zero. Finally, we set $\mathcal{A}_{h}=\mathcal{M}_{h} \times \mathcal{N}_{h}$ and $\mathcal{A}_{h, 0}=\mathcal{M}_{h, 0} \times \mathcal{N}_{h, 0}$. We note that for linear boundary conditions and polygonal domains, we have that $\mathcal{M}_{h} \subset \mathcal{M}$ and $\mathcal{N}_{h} \subset \mathcal{N}$ for the conforming finite element spaces discussed in this paper.

For the nonconforming finite element spaces the energy functional (2.1) is not well-defined, since the interfacial energy term only makes sense element-wise. Hence, the corresponding energy in these cases is

$$
\mathcal{E}_{\kappa, \theta, P, h}(y, b)=\sum_{K} \int_{K}\left\{\kappa\left(\left|\nabla^{2} y\right|^{2}+2|\nabla b|^{2}\right)+\phi(\nabla y \mid b, \theta)-P y_{3}\left(y_{1,1} y_{2,2}-y_{1,2} y_{2,1}\right)\right\} \mathrm{d} x,
$$

where the sum is taken over all triangles $K$ in the triangulation of $\Omega$. We note that the nonconforming energy above relaxes the penalty for oscillations in the deformation gradient across element edges. The oscillations for convergent nonconforming methods must approximately cancel so as to give global accuracy [36]. We found that the nonconforming Zienkiewicz element gave similar results to the conforming reduced HCT element for the "tent" problem with the mesh sizes $h$ and strain gradient coefficient $\kappa$ described in Section 6 .

To simplify our notation, we denote by $u$ the pair $(y, b) \in \mathcal{A}_{h}$ and by $\mathcal{E}(u), u \in \mathcal{A}_{h}$, the energy functional to be minimized over the space $\mathcal{A}_{h}$. For a fixed temperature and pressure, we choose an initial approximation $u^{0} \in \mathcal{A}_{h}$ and determine an iteration sequence, $\left\{u^{n}\right\} \subset \mathcal{A}_{h}$, such that $u^{n}$ converge to a local minimum of $\mathcal{E}$ as $n \rightarrow \infty$. The (local) minima of $\mathcal{E}$ determine (meta-)stable states of the thin film. We perform a simple continuation in pressure (or temperature), starting from a state of the film that is physically reasonable. Controlling the step size in pressure (or temperature), we can argue that the continuation procedure models the quasi-static behavior of the thin film.

The iteration procedure goes as follows. Having computed $u^{n} \in \mathcal{A}_{h}$, we determine a gradient direction $g^{n} \in \mathcal{A}_{h, 0}$ (see Sect. 4.1 below). We then determine a search direction $d^{n} \in \mathcal{A}_{h, 0}$ (see Sect. 4.2) and compute $\rho_{n}>0$ such that

$$
\mathcal{E}\left(u^{n}+\rho_{n} d^{n}\right) \leq \mathcal{E}\left(u^{n}+\rho d^{n}\right)
$$

for all $\rho$ in a neighborhood of $\rho_{n}$. Finally, we set

$$
u^{n+1}=u^{n}+\rho_{n} d^{n}
$$

and continue this process until a stopping criterion has been satisfied.

\subsection{Gradient direction}

Denote the first variation of the energy $\mathcal{E}(u)$ in the direction $v \in \mathcal{A}_{h, 0}$ by $\delta \mathcal{E}(u ; v)$. Then the gradient direction $g^{n} \in \mathcal{A}_{h}$ can be computed as the $L^{2}(\Omega)$-projection of this quantity, namely,

$$
\int_{\Omega} g^{n} \cdot v \mathrm{~d} x=\delta \mathcal{E}\left(u^{n} ; v\right) \quad \text { for all } v \in \mathcal{A}_{h, 0} .
$$

(See, for example, [20].) This way of computing $g^{n}$ results in a matrix system with a well-conditioned matrix, the mass matrix. However, this matrix still needs to be inverted, at least approximately. 
A computationally more efficient way is to project the first variation into the finite element space in the following way

$$
\hat{g}^{n}=\sum_{i=1}^{N} \delta \mathcal{E}\left(u^{n} ; v_{i}\right) v_{i}
$$

where $N=\operatorname{dim} \mathcal{A}_{h, 0}$ and the $v_{i}, i=1, \ldots, N$, form a basis of $\mathcal{A}_{h, 0}$. We can easily see that $-\hat{g}^{n}$ is a descent direction: letting $v=\sum_{i=1}^{N} c_{i} v_{i}$, we have for $\varepsilon>0$,

$$
\mathcal{E}\left(u^{n}-\varepsilon v\right)=\mathcal{E}\left(u^{n}\right)-\varepsilon \sum_{i=1}^{N} c_{i} \delta \mathcal{E}\left(u^{n} ; v_{i}\right)+o(\varepsilon) \quad \text { as } \varepsilon \rightarrow 0 .
$$

Hence, choosing $c_{i}=\delta \mathcal{E}\left(u^{n} ; v_{i}\right)$ defines a direction $v=\hat{g}^{n}$ such that $\mathcal{E}\left(u^{n}-\varepsilon v\right)<\mathcal{E}\left(u^{n}\right)$ for $\varepsilon$ positive and sufficiently small. Note that to determine $\hat{g}^{n}$, we only have to compute the values $\delta \mathcal{E}\left(u^{n} ; v_{i}\right.$ ) (which are the same as those in the right-hand side of the matrix system corresponding to (4.3)), because the mass matrix is replaced by the identity matrix. We have successfully used the computation of $\hat{g}^{n}$ as in (4.4) in our code.

\subsection{Search direction}

Having computed $g^{n}$, we need to choose the search direction $d^{n}$ for the univariate optimization (4.1). The simplest way is to set $d^{n}=-g^{n}$, corresponding to the steepest descent algorithm. We use variants of the conjugate gradient method to obtain greater efficiency. The Fletcher-Reeves variant $[28,45]$ starts with an initial guess, $u^{0} \in \mathcal{A}_{h}$, and an initial search direction $d^{0}=-g^{0} \in \mathcal{A}_{h, 0}$ is computed. After $u^{n} \in \mathcal{A}_{h}$ and $d^{n} \in \mathcal{A}_{h, 0}$ have been computed, we perform the line search as in (4.1) and $u^{n+1}$ is computed as in (4.2). We then compute the new gradient, $g^{n+1} \in \mathcal{A}_{h, 0}$, and a new search direction, $d^{n+1} \in \mathcal{A}_{h, 0}$, by

$$
d^{n+1}=-g^{n+1}+\lambda_{n} d^{n}
$$

where

$$
\lambda_{n}=\frac{\delta \mathcal{E}\left(u^{n+1} ; g^{n+1}\right)}{\delta \mathcal{E}\left(u^{n} ; g^{n}\right)} .
$$

The algorithm we choose for the univariate minimization (4.1) is the quadratic fit method [39]. This algorithm does not require evaluations of the derivatives of the function to be minimized, only the function itself. The local convergence of this method is super-linear, but to ensure global convergence the algorithm is usually modified and can, in the worst case, degenerate to linear convergence.

Finally, when the norm of the gradient reaches a given tolerance, the algorithm is stopped.

\subsection{Quadrature rules for the triangular elements}

In this section, we describe the quadrature rules that we use to approximate the integrals in the total energy. Clearly, different quadrature rules are needed for different terms and different elements. We do not discuss those elements or terms in the energy functional for which a low order quadrature rule will suffice (such as the $\mathcal{P}_{1}$ elements for $b$ or the interfacial energy term for the quadratic Morley element), but we mention those where higher-order quadrature rules are needed.

We have not established the order of convergence of the finite element methods (see Sect. 5 for obtained results). Therefore, we have not been able to study the effect of numerical quadrature on the convergence and determine the optimal quadrature rules, that is, those of the lowest possible order preserving the order of convergence. Hence, our choice was to integrate the interfacial energy terms exactly while using some higherorder rules for the other two terms. 


\subsubsection{The Zienkiewicz triangle (and the Argyris triangle)}

The interfacial energy term is the integral of a polynomial of degree 2 , therefore the quadrature rule utilizing only the values at the midpoints of the edges of the triangle with weights $1 / 3$ gives the exact value of the integral [17].

For the other lower-order terms, that is, for the elastic energy term and the pressure term, we use the 12-point Gaussian rule used, for example, in the software package Modulef for problems solved using the Argyris triangle (see also [24]). It is exact for polynomials in the space $\mathcal{P}_{6}$.

We also used this rule for all three terms in the energy functional when using the Argyris triangle.

\subsubsection{The reduced Hsieh-Clough-Tocher triangle (and the Morley triangle)}

Since the space $\mathcal{P}$ for this element is a space of functions that are polynomials on subtriangles $K_{i}$ of $K$, the quadrature rules should be applied to integrals over $K_{i}$ rather than $K$. On each $K_{i}$, the functions of $\mathcal{P}$ are cubic polynomials and hence the interfacial energy term is an integral of a polynomial of degree 2 . Therefore, we can use the same 3-point rule as for the interfacial energy term with the Zienkiewicz triangle.

For the elastic energy term and the pressure term, we use the 7-point Gaussian rule. This rule is exact for polynomials of degree 5. Note that this results in 21 nodes for each triangle $K$ rather than 36 had we used the 12-point quadrature rule described above in Section 4.3.1. Also note that the pressure term is an integral of a polynomial of degree 7 . However, to integrate this term exactly would require an excessive number of quadrature nodes (for example, the 13-point rule from [24] would require 39 quadrature nodes).

We also use the same 7-point quadrature rule for the elastic energy term and the pressure term when using the Morley element. Note that for this element the pressure term is an integral of a polynomial of degree 4, so we could conceivably use a lower-order quadrature rule for this term (see [24]).

\section{Theoretical Results}

In this section, we give some theoretical results concerning convergence of the conforming finite element methods. We show that for a global minimizer (or an isolated local minimizer) $u=(y, b)$ of the energy $(2.1)$ over the space $\mathcal{A}$ of admissible deformations there exists a sequence $\left\{u_{h}\right\} \in \mathcal{A}_{h}$ of global (or local) minimizers of the energy over the spaces $\mathcal{A}_{h}$ such that $\mathcal{E}_{\kappa, \theta, P}\left(u_{h}\right) \rightarrow \mathcal{E}_{\kappa, \theta, P}(u)$ and $u_{h} \rightarrow u$ in $H^{2}\left(\Omega ; \mathbb{R}^{3}\right) \times H^{1}\left(\Omega ; \mathbb{R}^{3}\right)$ as $h$, the diameter of the largest element $K$ in the triangulation of $\Omega$, goes to zero. We first give a result on the continuity and coercivity of the energy $\mathcal{E}_{\kappa, \theta, P}(y, b)$ for fixed parameters $\kappa>0, \theta \in \mathbb{R}$, and $P \geq 0$.

Lemma 5.1. We assume that the energy density, $\phi(F, \theta)$, is continuous in $F \in \mathbb{R}^{3 \times 3}$ and that for any $\theta \in \mathbb{R}$ there exist constants $c_{1}, c_{2}>0$ and $3<q_{1} \leq q_{2}$ such that

$$
c_{1}\left(|F|^{q_{1}}-1\right) \leq \phi(F, \theta) \leq c_{2}\left(|F|^{q_{2}}+1\right) \quad \text { for all } F \in \mathbb{R}^{3 \times 3} .
$$

Then the energy, $\mathcal{E}_{\kappa, \theta, P}(y, b)$, is weakly lower semi-continuous and strongly continuous on $H^{2}\left(\Omega ; \mathbb{R}^{3}\right) \times H^{1}\left(\Omega ; \mathbb{R}^{3}\right)$. Furthermore, there exists a positive constant $c_{3}$ and a constant $c_{4}$ such that

$$
\mathcal{E}_{\kappa, \theta, P}(y, b) \geq c_{3}\left(\|y\|_{H^{2}\left(\Omega ; \mathbb{R}^{3}\right)}^{2}+\|b\|_{H^{1}\left(\Omega ; \mathbb{R}^{3}\right)}^{2}\right)-c_{4}
$$

for all $(y, b) \in \mathcal{A}$.

Finally, if in addition $\phi(\cdot, \theta)$ is differentiable for every $\theta \in \mathbb{R}$ and there exist constants $c_{5}>0$ and $q_{3} \geq 0$ such that

$$
\left|\frac{\partial \phi}{\partial F}(F, \theta)\right| \leq c_{5}\left(|F|^{q_{3}}+1\right) \quad \text { for all } F \in \mathbb{R}^{3 \times 3}
$$


then the energy functional, $\mathcal{E}_{\kappa, \theta, P}: H^{2}\left(\Omega ; \mathbb{R}^{3}\right) \times H^{1}\left(\Omega ; \mathbb{R}^{3}\right) \rightarrow \mathbb{R}$, is Gâteaux differentiable and

$$
\begin{aligned}
\delta \mathcal{E}_{\kappa, \theta, P}((y, b) ;(v, c))= & \int_{\Omega} \kappa\left\{2\left(\nabla^{2} y\right) \cdot\left(\nabla^{2} v\right)+4(\nabla b) \cdot(\nabla c)\right\} \mathrm{d} x+\int_{\Omega} \frac{\partial \phi}{\partial F}(\nabla y \mid b, \theta) \cdot(\nabla v \mid c) \mathrm{d} x \\
& -P \int_{\Omega} v_{3}\left(y_{1,1} y_{2,2}-y_{1,2} y_{2,1}\right) \mathrm{d} x-P \int_{\Omega} y_{3}\left(v_{1,1} y_{2,2}-v_{1,2} y_{2,1}\right) \mathrm{d} x \\
& -P \int_{\Omega} y_{3}\left(y_{1,1} v_{2,2}-y_{1,2} v_{2,1}\right) \mathrm{d} x
\end{aligned}
$$

for all $(y, b),(v, c) \in H^{2}\left(\Omega ; \mathbb{R}^{3}\right) \times H^{1}\left(\Omega ; \mathbb{R}^{3}\right)$.

Proof. It is easily seen that

$$
\int_{\Omega} \kappa\left(\left|\nabla^{2} y\right|^{2}+2|\nabla b|^{2}\right) \mathrm{d} x
$$

is weakly lower semi-continuous and strongly continuous on $H^{2}\left(\Omega ; \mathbb{R}^{3}\right) \times H^{1}\left(\Omega ; \mathbb{R}^{3}\right)$.

From the Sobolev embedding theorem [1], we have that

$$
H^{2}\left(\Omega ; \mathbb{R}^{3}\right) \times H^{1}\left(\Omega ; \mathbb{R}^{3}\right) \subset W^{1, r}\left(\Omega ; \mathbb{R}^{3}\right) \times L^{r}\left(\Omega ; \mathbb{R}^{3}\right)
$$

is a compact embedding for any $1 \leq r<\infty$.

From the bounds for $\phi(F, \theta)$ in (5.1) and the embedding (5.5), we have by a generalization of the dominated convergence theorem [47, page 92] that

$$
\int_{\Omega} \phi(\nabla y \mid b, \theta) \mathrm{d} x
$$

is weakly continuous on $H^{2}\left(\Omega ; \mathbb{R}^{3}\right) \times H^{1}\left(\Omega ; \mathbb{R}^{3}\right)$.

Finally, we have by the identity

$$
A_{2} B_{2} C_{2}-A_{1} B_{1} C_{1}=\left(A_{2}-A_{1}\right) B_{2} C_{2}+A_{1}\left(B_{2}-B_{1}\right) C_{2}+A_{1} B_{1}\left(C_{2}-C_{1}\right),
$$

Hölder's inequality, and the embedding (5.5) that the term

$$
\int_{\Omega} y_{3}\left(y_{1,1} y_{2,2}-y_{1,2} y_{2,1}\right) \mathrm{d} x
$$

is weakly continuous on $H^{2}\left(\Omega ; \mathbb{R}^{3}\right) \times H^{1}\left(\Omega ; \mathbb{R}^{3}\right)$. Thus, we can conclude that the energy, $\mathcal{E}_{\kappa, \theta, P}(y, b)$, is weakly lower semi-continuous and strongly continuous on $H^{2}\left(\Omega ; \mathbb{R}^{3}\right) \times H^{1}\left(\Omega ; \mathbb{R}^{3}\right)$.

To prove the coercivity result (5.2), we observe by Hölder's inequality, the boundary condition (2.2), and the Poincaré inequality that there exists a positive constant $c_{6}$ and a constant $c_{7}$ such that

$$
\left|\int_{\Omega} y_{3}\left(y_{1,1} y_{2,2}-y_{1,2} y_{2,1}\right) \mathrm{d} x\right| \leq\left(\int_{\Omega}\left|y_{3}\right|^{3} \mathrm{~d} x\right)^{1 / 3}\left(\int_{\Omega}|\nabla y|^{3} \mathrm{~d} x\right)^{2 / 3} \leq c_{6} \int_{\Omega}|\nabla y|^{3} \mathrm{~d} x+c_{7}
$$

for all $y \in W^{1,3}\left(\Omega ; \mathbb{R}^{3}\right.$ ) (and hence for all $y \in H^{2}\left(\Omega ; \mathbb{R}^{3}\right)$ by $(5.5)$ ). It follows from the lower bound for $\phi(F, \theta)$ in (5.1) that since $q_{1}>3$ there exists a constant $c_{8}$, depending on $c_{1}$ and $c_{6} P+1$, such that

$$
\phi(F, \theta) \geq\left(c_{6} P+1\right)|F|^{3}-c_{8} \quad \text { for all } F \in \mathbb{R}^{3 \times 3} .
$$


Hence, we have from (5.6) and (5.7)

$$
\int_{\Omega} \phi(\nabla y \mid b, \theta)-P y_{3}\left(y_{1,1} y_{2,2}-y_{1,2} y_{2,1}\right) \mathrm{d} x \geq \int_{\Omega}|\nabla y|^{3} \mathrm{~d} x-c_{7} P-c_{8} \text { meas } \Omega .
$$

We thus obtain that

$$
\begin{aligned}
\mathcal{E}_{\kappa, \theta, P}(y, b) & =\int_{\Omega}\left\{\kappa\left(\left|\nabla^{2} y\right|^{2}+2|\nabla b|^{2}\right)+\phi(\nabla y \mid b, \theta)-P y_{3}\left(y_{1,1} y_{2,2}-y_{1,2} y_{2,1}\right)\right\} \mathrm{d} x \\
& \geq \int_{\Omega} \kappa\left(\left|\nabla^{2} y\right|^{2}+2|\nabla b|^{2}\right) \mathrm{d} x+\int_{\Omega}|\nabla y|^{3} \mathrm{~d} x-c_{7} P-c_{8} \text { meas } \Omega
\end{aligned}
$$

for $(y, b) \in H^{2}\left(\Omega ; \mathbb{R}^{3}\right) \times H^{1}\left(\Omega ; \mathbb{R}^{3}\right)$. The coercivity result $(5.2)$ for $(y, b) \in \mathcal{A}$ then follows by the Poincaré inequality.

Finally, to prove that the energy, $\mathcal{E}_{\kappa, \theta, P}$, is Gâteaux differentiable and that $(5.4)$ holds, it is enough to show that the elastic energy term is Gâteaux differentiable and that, for a fixed $\varepsilon>0$,

$$
\frac{\mathrm{d}}{\mathrm{d} s} \int_{\Omega} \phi\left(w_{1}(x)+s w_{2}(x)\right) \mathrm{d} x=\int_{\Omega} \frac{\partial \phi}{\partial F}\left(w_{1}(x)+s w_{2}(x)\right) \cdot w_{2}(x) \mathrm{d} x
$$

for $s \in(-\varepsilon, \varepsilon)$ and $w_{1}, w_{2} \in H^{1}\left(\Omega ; \mathbb{R}^{3 \times 3}\right)$. However, this follows from [25, Theorem 2.27], since we have by (5.3)

$$
\begin{aligned}
\left|\frac{\partial \phi}{\partial F}\left(w_{1}(x)+s w_{2}(x)\right) \cdot w_{2}(x)\right| & \leq c_{5}\left(\left|w_{1}(x)+s w_{2}(x)\right|^{q_{3}}+1\right)\left|w_{2}(x)\right| \\
& \leq c_{9}\left|w_{1}(x)\right|^{q_{3}+1}+c_{10}\left|w_{2}(x)\right|^{q_{3}+1}+c_{11}\left|w_{2}(x)\right|
\end{aligned}
$$

for some constants $c_{9}, c_{10}, c_{11}$, where the right-hand side is independent of $s$ and belongs to $L^{1}(\Omega ; \mathbb{R})$ due to the embedding (5.5).

Remark 5.1. We note that we can take $c_{7}=0$ for the boundary conditions (2.5). We also note that the constant $c_{4}$ in (5.2) depends on $q_{1}, c_{1}$, and the pressure $P$. Using the same proof as above, the coercivity result (5.2) can be extended to the case $q_{1}=3$ for $P$ such that $c_{5} P<c_{1}$.

We next give a result for the existence of a global minimizer $u=(y, b) \in \mathcal{A}$ of the energy (2.1) for fixed parameters $\kappa>0, \theta \in \mathbb{R}$, and $P \geq 0$.

Lemma 5.2. Let $\kappa>0, \theta \in \mathbb{R}$ and $P \geq 0$ be given. Assume that the growth condition (5.1) holds. Then there exists a global minimizer $u=(y, b) \in \mathcal{A}$ of the total energy (2.1).

Proof. We have by the coercivity inequality (5.2) that the energy $\mathcal{E}_{\kappa, \theta, P}$ is bounded below for $(y, b) \in \mathcal{A}$. Consider an energy minimizing sequence $\left\{\left(y_{n}, b_{n}\right)\right\} \subset \mathcal{A}$. By the coercivity inequality (5.2), this sequence is bounded in $H^{2}\left(\Omega ; \mathbb{R}^{3}\right) \times H^{1}\left(\Omega ; \mathbb{R}^{3}\right)$. Considering a weakly convergent subsequence, the existence of a minimizer of $\mathcal{E}_{\kappa, \theta, P}$ over $\mathcal{A}$ then follows from the weak lower semi-continuity of $\mathcal{E}_{\kappa, \theta, P}$ over $H^{2}\left(\Omega ; \mathbb{R}^{3}\right) \times H^{1}\left(\Omega ; \mathbb{R}^{3}\right)$ and the weak closedness of $\mathcal{A}$ in $H^{2}\left(\Omega ; \mathbb{R}^{3}\right) \times H^{1}\left(\Omega ; \mathbb{R}^{3}\right)$.

Remark 5.2. We note that the energy density defined in (6.6) below satisfies (5.1). We also remark that using the same argument as in the proof of Lemma 5.2 we can show the existence of a minimizer of the total energy (2.1) over any nonempty, weakly closed subset of $\mathcal{A}$. Also, the finite dimensionality of $\mathcal{A}_{h}$ and the coercivity inequality (5.2) ensures the existence of minimizers of the total energy (2.1) over the finite element spaces $\mathcal{A}_{h}$ for any $h>0$.

Our next result gives the strong convergence of minimizing sequences of the energy. 
Theorem 5.1. Let $\kappa>0, \theta \in \mathbb{R}$ and $P \geq 0$ be given. Assume that the growth condition (5.1) holds. Let $\left\{\left(y_{n}, b_{n}\right)\right\}$ be a minimizing sequence in $\mathcal{A}$ so that

$$
\mathcal{E}_{\kappa, \theta, P}\left(y_{n}, b_{n}\right) \rightarrow \min _{(\tilde{y}, \tilde{b}) \in \mathcal{A}} \mathcal{E}_{\kappa, \theta, P}(\tilde{y}, \tilde{b}) \quad \text { as } h \rightarrow 0
$$

Then there exists a subsequence of $\left\{\left(y_{n}, b_{n}\right)\right\}$, not relabeled, such that as $n \rightarrow \infty$

$$
\begin{aligned}
& y_{n} \rightarrow y \quad \text { in } H^{2}\left(\Omega ; \mathbb{R}^{3}\right), \\
& b_{n} \rightarrow b \quad \text { in } H^{1}\left(\Omega ; \mathbb{R}^{3}\right),
\end{aligned}
$$

where $u=(y, b)$ minimizes the total energy (2.1) over $\mathcal{A}$. that

If the energy (2.1) has a unique minimizer $u=(y, b) \in \mathcal{A}$, then we have for the complete sequence $\left\{\left(y_{n}, b_{n}\right)\right\}$

$$
\begin{aligned}
& y_{n} \rightarrow y \quad \text { in } H^{2}\left(\Omega ; \mathbb{R}^{3}\right), \\
& b_{n} \rightarrow b \quad \text { in } H^{1}\left(\Omega ; \mathbb{R}^{3}\right) .
\end{aligned}
$$

Proof. Recall from the proof of Lemma 5.2 that the fact that $\left\{\left(y_{n}, b_{n}\right)\right\}$ is an energy minimizing sequence in $\mathcal{A}$ implies that there exists $u=(y, b) \in \mathcal{A}$ and a subsequence of $\left\{\left(y_{n}, b_{n}\right)\right\}$, not relabeled, such that

$$
\mathcal{E}_{\kappa, \theta, P}(y, b)=\min _{(\tilde{y}, \tilde{b}) \in \mathcal{A}} \mathcal{E}_{\kappa, \theta, P}(\tilde{y}, \tilde{b})=\lim _{n \rightarrow \infty} \mathcal{E}_{\kappa, \theta, P}\left(y_{n}, b_{n}\right)
$$

and

$$
\begin{array}{ll}
y_{n} \rightarrow y & \text { in } H^{2}\left(\Omega ; \mathbb{R}^{3}\right), \\
b_{n} \rightarrow b & \text { in } H^{1}\left(\Omega ; \mathbb{R}^{3}\right) .
\end{array}
$$

From the proof of Lemma 5.1 we have that the elastic energy term and the pressure term are weakly continuous over $H^{2}\left(\Omega ; \mathbb{R}^{3}\right) \times H^{1}\left(\Omega ; \mathbb{R}^{3}\right)$. Hence, it follows that

$$
\begin{aligned}
\limsup _{n \rightarrow \infty} & \mathcal{E}_{\kappa, \theta, P}\left(y_{n}, b_{n}\right)-\mathcal{E}_{\kappa, \theta, P}(y, b) \\
\quad= & \limsup _{n \rightarrow \infty} \int_{\Omega} \kappa\left\{\left|\nabla^{2}\left(y_{n}-y\right)\right|^{2}+2\left(\nabla^{2} y\right) \cdot \nabla^{2}\left(y_{n}-y\right)+2\left|\nabla\left(b_{n}-b\right)\right|^{2}+4(\nabla b) \cdot \nabla\left(b_{n}-b\right)\right\} \mathrm{d} x \\
\quad= & \limsup _{n \rightarrow \infty} \int_{\Omega} \kappa\left\{\left|\nabla^{2}\left(y_{n}-y\right)\right|^{2}+2\left|\nabla\left(b_{n}-b\right)\right|^{2}\right\} \mathrm{d} x .
\end{aligned}
$$

Therefore, since we can conclude from (5.10) that

$$
\limsup _{n \rightarrow \infty} \int_{\Omega} \kappa\left\{\left|\nabla^{2}\left(y_{n}-y\right)\right|^{2}+2\left|\nabla\left(b_{n}-b\right)\right|^{2}\right\} \mathrm{d} x=0
$$

we have established the strong convergence in the $H^{2}\left(\Omega ; \mathbb{R}^{3}\right) \times H^{1}\left(\Omega ; \mathbb{R}^{3}\right)$ topology.

In our analysis below, we shall assume that the deformation $y \in \mathcal{M}$ is approximated by the reduced HsiehClough-Tocher element $[9,17]$, although the analysis applies to other conforming methods and even to some nonconforming methods. We shall assume that $b \in \mathcal{N}$ is approximated by the continuous, piecewise linear element. We also assume that the boundary conditions on $\mathcal{A}$ are compatible with the finite element approximation in the sense that $\mathcal{A}_{h} \subset \mathcal{A}$ for all $h$, where $h$ denotes the maximum of the diameters of the elements of 
a triangulation of $\Omega$. (For the linear boundary conditions of the tent problem $(2.5)$ with $\Omega=(0,1) \times(0,1)$ this is the case.) So, in what follows $\mathcal{A}_{h}=\mathcal{M}_{h} \times \mathcal{N}_{h}$, where $\mathcal{M}_{h}$ is the reduced Hsieh-Clough-Tocher finite element space and $\mathcal{N}_{h}$ is the continuous, piecewise linear finite element space.

We assume that a quasi-regular family of triangulations $\left\{\tau_{h}\right\}$ of $\Omega$ is given, that is, there exists a constant $\sigma$ such that, for all $K$ in each triangulation and for all triangulations of the family,

$$
h_{K} \leq \sigma \rho_{K},
$$

where $h_{K}$ denotes the diameter of $K$ and $\rho_{K}$ the supremum of the diameters of balls contained in $K$. We also assume that $h=\max _{K \in \tau_{h}} h_{K}$. We then have the following result for the density of $\mathcal{A}_{h}=\mathcal{M}_{h} \times \mathcal{N}_{h}$ in $\mathcal{A}=\mathcal{M} \times \mathcal{N}$ as $h \rightarrow 0$ (see Th. 3.2.3 and 6.1.7 in [17]).

Lemma 5.3. Assume that a quasi-regular family of triangulations $\left\{\tau_{h}\right\}$ of $\Omega$ is given. Then for any $\tilde{u}=(\tilde{y}, \tilde{b}) \in$ $\mathcal{A}$, we have that

$$
\lim _{h \rightarrow 0} \inf _{\left(\tilde{y}_{h}, \tilde{b}_{h}\right) \in \mathcal{A}_{h}}\left(\left\|\tilde{y}-\tilde{y}_{h}\right\|_{H^{2}\left(\Omega ; \mathbb{R}^{3}\right)}+\left\|\tilde{b}-\tilde{b}_{h}\right\|_{H^{1}\left(\Omega ; \mathbb{R}^{3}\right)}\right)=0 .
$$

The next result on the convergence of the energy of the finite element approximations $u_{h}$ of $u$ follows from the above result and from the continuity of $\mathcal{E}_{\kappa, \theta, P}$ over $H^{2}\left(\Omega ; \mathbb{R}^{3}\right) \times H^{1}\left(\Omega ; \mathbb{R}^{3}\right)$.

Theorem 5.2. Let $\kappa>0, \theta \in \mathbb{R}$ and $P \geq 0$ be given. Assume that the growth condition (5.1) holds. Then

$$
\min _{\left(\tilde{y}_{h}, \tilde{b}_{h}\right) \in \mathcal{A}_{h}} \mathcal{E}_{\kappa, \theta, P}\left(\tilde{y}_{h}, \tilde{b}_{h}\right) \rightarrow \min _{(\tilde{y}, \tilde{b}) \in \mathcal{A}} \mathcal{E}_{\kappa, \theta, P}(\tilde{y}, \tilde{b}) \quad \text { as } h \rightarrow 0 .
$$

The following result on the strong convergence of global minimizers of finite element approximations, $\left(y_{h}, b_{h}\right) \in$ $\mathcal{A}_{h}$, follows as a corollary of Theorems 5.1 and 5.2.

Corollary 5.1. Let $\kappa>0, \theta \in \mathbb{R}$ and $P \geq 0$ be given. Assume that the growth condition (5.1) holds. Let $\left\{\left(y_{n}, b_{n}\right)\right\}$ be global minimizers of the energy over $\mathcal{A}_{h}$ for a family of quasi-regular triangulations. Then there exists a subsequence of $\left\{\left(y_{n}, b_{n}\right)\right\}$, not relabeled, such that as $h \rightarrow 0$

$$
\begin{aligned}
& y_{h} \rightarrow y \quad \text { in } H^{2}\left(\Omega ; \mathbb{R}^{3}\right), \\
& b_{h} \rightarrow b \quad \text { in } H^{1}\left(\Omega ; \mathbb{R}^{3}\right),
\end{aligned}
$$

where $(y, b)$ minimizes the total energy (2.1) over $\mathcal{A}$.

If the energy (2.1) has a unique minimizer $(y, b) \in \mathcal{A}$, then we have for the complete sequence $\left\{\left(y_{n}, b_{n}\right)\right\}$ that

$$
\begin{aligned}
& y_{h} \rightarrow y \quad \text { in } H^{2}\left(\Omega ; \mathbb{R}^{3}\right), \\
& b_{h} \rightarrow b \quad \text { in } H^{1}\left(\Omega ; \mathbb{R}^{3}\right) .
\end{aligned}
$$

We now recall the rate of approximation of $(\tilde{y}, \tilde{b}) \in H^{3}\left(\Omega ; \mathbb{R}^{3}\right) \times H^{2}\left(\Omega ; \mathbb{R}^{3}\right) \cap \mathcal{A}$ by the finite element functions $\left(y_{h}, b_{h}\right) \in \mathcal{A}_{h}=\mathcal{M}_{h} \times \mathcal{N}_{h} \subset \mathcal{A}[9,17]$.

Lemma 5.4. Assume that a quasi-regular family of triangulations $\left\{\tau_{h}\right\}$ of $\Omega$ is given. Then there exists a constant $C$, independent of $h$, such that

$$
\begin{array}{ll}
\inf _{\tilde{y}_{h} \in \mathcal{M}_{h}}\left\{\left\|\tilde{y}-\tilde{y}_{h}\right\|_{0}+h\left\|\tilde{y}-\tilde{y}_{h}\right\|_{1}+h^{2}\left\|\tilde{y}-\tilde{y}_{h}\right\|_{2}\right\} \leq C h^{3}|\tilde{y}|_{3}, & \tilde{y} \in H^{3}\left(\Omega ; \mathbb{R}^{3}\right) \cap \mathcal{M}, \\
\inf _{\tilde{b}_{h} \in \mathcal{N}_{h}}\left\{\left\|\tilde{b}-\tilde{b}_{h}\right\|_{0}+h\left\|\tilde{b}-\tilde{b}_{h}\right\|_{1}\right\} \leq C h^{2}|\tilde{b}|_{2}, & \tilde{b} \in H^{2}\left(\Omega ; \mathbb{R}^{3}\right) \cap \mathcal{N},
\end{array}
$$

where $\|\cdot\|_{k}$ denotes the $H^{k}(\Omega)$-norm and $|\cdot|_{k}$ the $H^{k}(\Omega)$-seminorm. 
For $(\tilde{y}, \tilde{b}) \in \mathcal{M} \times \mathcal{N}$ we can define the projection $\Pi_{h}(\tilde{y}, \tilde{b})=\left(\Pi_{h}^{y} \tilde{y}, \Pi_{h}^{b} \tilde{b}\right) \in \mathcal{M}_{h} \times \mathcal{N}_{h}$ by

$$
\begin{aligned}
& \left\{\left\|\tilde{y}-\Pi_{h}^{y} \tilde{y}\right\|_{0}+h\left\|\tilde{y}-\Pi_{h}^{y} \tilde{y}\right\|_{1}+h^{2}\left\|\tilde{y}-\Pi_{h}^{y} \tilde{y}\right\|_{2}\right\}=\inf _{\tilde{y}_{h} \in \mathcal{M}_{h}}\left\{\left\|\tilde{y}-\tilde{y}_{h}\right\|_{0}+h\left\|\tilde{y}-\tilde{y}_{h}\right\|_{1}+h^{2}\left\|\tilde{y}-\tilde{y}_{h}\right\|_{2}\right\}, \\
& \left\{\left\|\tilde{b}-\Pi_{h}^{b} \tilde{b}\right\|_{0}+h\left\|\tilde{b}-\Pi_{h}^{b} \tilde{b}\right\|_{1}\right\}=\inf _{\tilde{b}_{h} \in \mathcal{N}_{h}}\left\{\left\|\tilde{b}-\tilde{b}_{h}\right\|_{0}+h\left\|\tilde{b}-\tilde{b}_{h}\right\|_{1}\right\} .
\end{aligned}
$$

By classical bootstrap arguments, a minimizer $u=(y, b) \in \mathcal{A}$ of the total energy (2.1) will be smooth in the interior of $\Omega$ if $\phi$ is smooth and satisfies the growth conditions (5.1) [26,27]. For appropriate boundary conditions $(y, b)=(\hat{y}, \hat{b})$ on $\Gamma$, we can expect that $(y, b) \in H^{3}\left(\Omega ; \mathbb{R}^{3}\right) \times H^{2}\left(\Omega ; \mathbb{R}^{3}\right)$. If the minimizer $(y, b)$ has reduced regularity, then the following proof will give a reduced rate of convergence.

Theorem 5.3. We assume that a quasi-regular family of triangulations $\left\{\tau_{h}\right\}$ of $\Omega$ is given. We also assume that $\phi$ satisfies the growth condition (5.1) and that it is differentiable and satisfies (5.3). We assume further that a minimizer $u=(y, b) \in \mathcal{A}$ of the total energy (2.1) (whose existence is guaranteed by Lem. 5.2) satisfies the regularity assumption

$$
(y, b) \in H^{3}\left(\Omega ; \mathbb{R}^{3}\right) \times H^{2}\left(\Omega ; \mathbb{R}^{3}\right) .
$$

Then there exists a constant $C>0$, independent of $h$, such that

$$
0 \leq \min _{\left(\tilde{y}_{h}, \tilde{b}_{h}\right) \in \mathcal{A}_{h}} \mathcal{E}_{\kappa, \theta, P}\left(\tilde{y}_{h}, \tilde{b}_{h}\right)-\min _{(\tilde{y}, \tilde{b}) \in \mathcal{A}} \mathcal{E}_{\kappa, \theta, P}(\tilde{y}, \tilde{b}) \leq C h^{2}
$$

Proof. The first inequality in (5.12) is trivial since $\mathcal{A}_{h} \subset \mathcal{A}$. For the second one, define $\left(\Pi_{h}^{y} y, \Pi_{h}^{b} b\right)$ by (5.11). We then have that

$$
\min _{\left(\tilde{y}_{h}, \tilde{b}_{h}\right) \in \mathcal{A}_{h}} \mathcal{E}_{\kappa, \theta, P}\left(\tilde{y}_{h}, \tilde{b}_{h}\right)-\min _{(\tilde{y}, \tilde{b}) \in \mathcal{A}} \mathcal{E}_{\kappa, \theta, P}(\tilde{y}, \tilde{b}) \leq \mathcal{E}_{\kappa, \theta, P}\left(\Pi_{h}^{y} y, \Pi_{h}^{b} b\right)-\mathcal{E}_{\kappa, \theta, P}(y, b)
$$

since $(y, b) \in \mathcal{A}$ is a minimizer and $\left(\Pi_{h}^{y} y, \Pi_{h}^{b} b\right) \in \mathcal{A}_{h}$. We next define $\left(e_{h}^{y}, e_{h}^{b}\right)$ by $\left(e_{h}^{y}, e_{h}^{b}\right)=\left(\Pi_{h}^{y} y, \Pi_{h}^{b} b\right)-(y, b)$. Then

$$
\begin{aligned}
\mathcal{E}_{\kappa, \theta, P}\left(\Pi_{h}^{y} y, \Pi_{h}^{b} b\right)-\mathcal{E}_{\kappa, \theta, P}(y, b)= & \int_{\Omega} \kappa\left\{\left|\nabla^{2} e_{h}^{y}\right|^{2}+2\left(\nabla^{2} y\right) \cdot\left(\nabla^{2} e_{h}^{y}\right)+2\left|\nabla e_{h}^{b}\right|^{2}+4(\nabla b) \cdot\left(\nabla e_{h}^{b}\right)\right\} \mathrm{d} x \\
& +\int_{\Omega}\left[\phi\left(\nabla\left(y+e_{h}^{y}\right) \mid b+e_{h}^{b}, \theta\right)-\phi(\nabla y \mid b, \theta)\right] \mathrm{d} x \\
& -P \int_{\Omega}\left[\left(y_{3}+e_{h 3}^{y}\right)\left(\left(y_{1,1}+e_{h 1,1}^{y}\right)\left(y_{2,2}+e_{h 2,2}^{y}\right)-\left(y_{1,2}+e_{h 1,2}^{y}\right)\left(y_{2,1}+e_{h 2,1}^{y}\right)\right)\right] \mathrm{d} x \\
& +P \int_{\Omega} y_{3}\left(y_{1,1} y_{2,2}-y_{1,2} y_{2,1}\right) \mathrm{d} x .
\end{aligned}
$$


Since $(y, b)$ is a minimizer over $\mathcal{A}$ and since $\phi$ is differentiable and satisfies (5.3), the Euler-Lagrange equation (5.4) with $(v, c)=\left(e_{h}^{y}, e_{h}^{b}\right)$ is equal to 0 , and hence

$$
\begin{aligned}
\mathcal{E}_{\kappa, \theta, P}\left(\Pi_{h}^{y} y, \Pi_{h}^{b} b\right)-\mathcal{E}_{\kappa, \theta, P}(y, b)= & \int_{\Omega} \kappa\left\{\left|\nabla^{2} e_{h}^{y}\right|^{2}+2\left|\nabla e_{h}^{b}\right|^{2}\right\} \mathrm{d} x \\
& +\int_{\Omega}\left[\phi\left(\nabla\left(y+e_{h}^{y}\right) \mid b+e_{h}^{b}, \theta\right)-\phi(\nabla y \mid b, \theta)-\frac{\partial \phi}{\partial F}(\nabla y \mid b, \theta) \cdot\left(\nabla e_{h}^{y}, e_{h}^{b}\right)\right] \mathrm{d} x \\
& -P \int_{\Omega}\left(y_{3}+e_{h 3}^{y}\right)\left(e_{h 1,1}^{y} e_{h 2,2}^{y}-e_{h 1,2}^{y} e_{h 2,1}^{y}\right) \mathrm{d} x \\
& -P \int_{\Omega} e_{h 3}^{y}\left(e_{h 1,1}^{y} y_{2,2}+y_{1,1} e_{h 2,2}^{y}-e_{h 1,2}^{y} y_{2,1}-y_{1,2} e_{h 2,1}^{y}\right) \mathrm{d} x .
\end{aligned}
$$

Denoting now by $C$ a generic constant independent of $h$ (but depending on $(y, b)$ and $\Omega$ ), we find by using Lemma 5.4, the mean value theorem, the Cauchy-Schwarz inequality, and the growth assumption (5.3) that

$$
\int_{\Omega} \kappa\left\{\left|\nabla^{2} e_{h}^{y}\right|^{2}+2\left|\nabla e_{h}^{b}\right|^{2}\right\} \mathrm{d} x \leq C h^{2},
$$

and

$$
\int_{\Omega}\left|\phi\left(\nabla\left(y+e_{h}^{y}\right) \mid b+e_{h}^{b}, \theta\right)-\phi(\nabla y \mid b, \theta)-\frac{\partial \phi}{\partial F}(\nabla y \mid b, \theta) \cdot\left(\nabla e_{h}^{y}, e_{h}^{b}\right)\right| \mathrm{d} x \leq C h^{2} .
$$

From the embedding [1]

$$
H^{2}(\Omega) \subset \mathcal{C}(\bar{\Omega})
$$

and the fact that $\left\|e_{h}^{y}\right\|_{H^{2}\left(\Omega ; \mathbb{R}^{3}\right)} \rightarrow 0$ as $h \rightarrow 0$, we have

$$
\int_{\Omega}\left|\left(y_{3}+e_{h 3}^{y}\right)\left(e_{h 1,1}^{y} e_{h 2,2}^{y}-e_{h 1,2}^{y} e_{h 2,1}^{y}\right)\right| \mathrm{d} x \leq C \int_{\Omega}\left|\nabla e_{h}^{y}\right|^{2} \mathrm{~d} x \leq C h^{4} .
$$

From the fact that $y \in H^{3}\left(\Omega ; \mathbb{R}^{3}\right)$ we have

$$
\|\nabla y\|_{L^{\infty}\left(\Omega ; \mathbb{R}^{3 \times 2}\right)} \leq C\|y\|_{H^{3}\left(\Omega ; \mathbb{R}^{3}\right)},
$$

and hence, by the Cauchy-Schwarz inequality,

$$
\int_{\Omega}\left|e_{h 3}^{y}\left(e_{h 1,1}^{y} y_{2,2}+y_{1,1} e_{h 2,2}^{y}-e_{h 1,2}^{y} y_{2,1}-y_{1,2} e_{h 2,1}^{y}\right)\right| \mathrm{d} x \leq C\left\|e_{h}^{y}\right\|_{L^{2}\left(\Omega ; \mathbb{R}^{3}\right)}\left\|\nabla e_{h}^{y}\right\|_{L^{2}\left(\Omega ; \mathbb{R}^{3 \times 2}\right)} \leq C h^{5} .
$$

Adding all of the above terms establishes the claim of the theorem.

Remark 5.3. We note that we can obtain a weaker result under weaker assumptions. If we assume that $\phi$ satisfies a local Lipschitz condition of the form

$$
\left|\phi\left(F_{1}, \theta\right)-\phi\left(F_{2}, \theta\right)\right| \leq C\left|F_{1}-F_{2}\right|\left[1+\left(\left|F_{1}\right|+\left|F_{2}\right|\right)^{q_{3}}\right] \quad \text { for all } F_{1}, F_{2} \in \mathbb{R}^{3 \times 3},
$$

where $q_{3} \geq 0$ (instead of differentiability of $\phi$ and the growth condition (5.3)), then it is easy to see from (5.13) that

$$
0 \leq \min _{\left(\tilde{y}_{h}, \tilde{b}_{h}\right) \in \mathcal{A}_{h}} \mathcal{E}_{\kappa, \theta, P}\left(\tilde{y}_{h}, \tilde{b}_{h}\right)-\min _{(\tilde{y}, \tilde{b}) \in \mathcal{A}} \mathcal{E}_{\kappa, \theta, P}(\tilde{y}, \tilde{b}) \leq C h
$$


On the other hand, if the minimizer satisfies

$$
(y, b) \in H^{4}\left(\Omega ; \mathbb{R}^{3}\right) \times H^{3}\left(\Omega ; \mathbb{R}^{3}\right),
$$

and the space $\mathcal{M}$ is approximated by a finite element space $\mathcal{M}_{h} \subset \mathcal{M}$ such that piecewise cubic functions belong to $\mathcal{M}_{h}$ (for example, the full Hsieh-Clough-Tocher or the Argyris finite element space) and $\mathcal{N}$ is approximated by the continuous, piecewise quadratic finite element space $\mathcal{N}_{h}$, we obtain that the left-hand side of (5.14) is bounded by $C h^{4}$. Similarly, if the energy density, $\phi$, is twice differentiable everywhere with respect to $F \in \mathbb{R}^{3 \times 3}$, and the second derivative satisfies a growth condition similar to (5.3), the left-hand side of (5.15) is bounded by $C h^{6}$ and hence

$$
0 \leq \min _{\left(\tilde{y}_{h}, \tilde{b}_{h}\right) \in \mathcal{A}_{h}} \mathcal{E}_{\kappa, \theta, P}\left(\tilde{y}_{h}, \tilde{b}_{h}\right)-\min _{(\tilde{y}, \tilde{b}) \in \mathcal{A}} \mathcal{E}_{\kappa, \theta, P}(\tilde{y}, \tilde{b}) \leq C h^{4} .
$$

Remark 5.4. We note that if the global minimizer $(y, b)$ is sufficiently smooth and the determinant of $(\nabla y, b)$ is positive, then we can expect that $\left(\nabla \Pi_{h}^{y} y, \Pi_{h}^{b} b\right)$ has positive determinant and that the result of Theorem 5.3 holds when the spaces $\mathcal{A}$ and $\mathcal{A}_{h}$ are restricted to functions constrained by the requirement that $(\nabla y, b)$ has positive deformation gradient.

The above results can be extended to a statement about an isolated local energy minimizer $u=(y, b) \in \mathcal{A}$.

Theorem 5.4. Let $\kappa>0, \theta \in \mathbb{R}$ and $P \geq 0$ be given. Assume that the growth condition (5.1) holds. Let $u=(y, b) \in \mathcal{A}$ be an isolated local minimizer of the energy (2.1) in the $H^{2}\left(\Omega ; \mathbb{R}^{3}\right) \times H^{1}\left(\Omega ; \mathbb{R}^{3}\right)$ topology. Given a quasi-regular family of triangulations $\left\{\tau_{h}\right\}$ and the corresponding conforming finite element spaces $\mathcal{A}_{h}$ with $h$ sufficiently small, there exists a sequence of local minimizers $\left(y_{h}, b_{h}\right) \in \mathcal{A}_{h}$ of the energy (2.1) over the spaces $\mathcal{A}_{h}$ such that as $h \rightarrow 0$

$$
\begin{aligned}
& y_{h} \rightarrow y \quad \text { in } H^{2}\left(\Omega ; \mathbb{R}^{3}\right), \\
& b_{h} \rightarrow b \quad \text { in } H^{1}\left(\Omega ; \mathbb{R}^{3}\right) .
\end{aligned}
$$

Proof. Since $u=(y, b) \in \mathcal{A}$ is an isolated local minimizer of the energy (2.1), there exists an open ball $\mathcal{B} \subset H^{2}\left(\Omega ; \mathbb{R}^{3}\right) \times H^{1}\left(\Omega ; \mathbb{R}^{3}\right)$ containing $u$ such that

$$
\mathcal{E}_{\kappa, \theta, P}(y, b)<\mathcal{E}_{\kappa, \theta, P}(\tilde{y}, \tilde{b})
$$

for all $(\tilde{y}, \tilde{b}) \in \mathcal{A} \cap \overline{\mathcal{B}}$ such that $(\tilde{y}, \tilde{b}) \neq(y, b)$, where $\overline{\mathcal{B}}$ denotes the closure of $\mathcal{B}$ in $H^{2}\left(\Omega ; \mathbb{R}^{3}\right) \times H^{1}\left(\Omega ; \mathbb{R}^{3}\right)$. It follows from Lemma 5.3 that for any quasi-regular family of triangulations $\left\{\tau_{h}\right\}$ there exist $\left(\tilde{y}_{h}, \tilde{b}_{h}\right) \in \mathcal{A}_{h}$ such that

$$
\left(\tilde{y}_{h}, \tilde{b}_{h}\right) \rightarrow(y, b) \quad \text { in } H^{2}\left(\Omega ; \mathbb{R}^{3}\right) \times H^{1}\left(\Omega ; \mathbb{R}^{3}\right) \quad \text { as } h \rightarrow 0,
$$

and hence the finite element spaces $\mathcal{A}_{h}$ satisfy $\mathcal{A}_{h} \cap \overline{\mathcal{B}} \neq \emptyset$ for $h$ sufficiently small. Let $\left(y_{h}, b_{h}\right) \in \mathcal{A}_{h} \cap \overline{\mathcal{B}}$ be such that

$$
\mathcal{E}_{\kappa, \theta, P}\left(y_{h}, b_{h}\right)=\min _{\left(\tilde{y}_{h}, \tilde{b}_{h}\right) \in \mathcal{A}_{h} \cap \overline{\mathcal{B}}} \mathcal{E}_{\kappa, \theta, P}\left(\tilde{y}_{h}, \tilde{b}_{h}\right) .
$$

From the strong continuity of the energy in $H^{2}\left(\Omega ; \mathbb{R}^{3}\right) \times H^{1}\left(\Omega ; \mathbb{R}^{3}\right)$ we have that

$$
\mathcal{E}_{\kappa, \theta, P}\left(\tilde{y}_{h}, \tilde{b}_{h}\right) \rightarrow \mathcal{E}_{\kappa, \theta, P}(y, b) \quad \text { as } h \rightarrow 0,
$$

and therefore it follows from the inequality $\mathcal{E}_{\kappa, \theta, P}(y, b) \leq \mathcal{E}_{\kappa, \theta, P}\left(y_{h}, b_{h}\right) \leq \mathcal{E}_{\kappa, \theta, P}\left(\tilde{y}_{h}, \tilde{b}_{h}\right)$ that

$$
\mathcal{E}_{\kappa, \theta, P}\left(y_{h}, b_{h}\right) \rightarrow \mathcal{E}_{\kappa, \theta, P}(y, b) \quad \text { as } h \rightarrow 0 .
$$


Since the sequence $\left\{\left(y_{h}, b_{h}\right)\right\}$ is bounded in $H^{2}\left(\Omega ; \mathbb{R}^{3}\right) \times H^{1}\left(\Omega ; \mathbb{R}^{3}\right)$, there exists a subsequence of $\left\{\left(y_{h}, b_{h}\right)\right\}$, not relabeled, and $(\tilde{y}, \tilde{b}) \in \mathcal{A}$ such that as $h \rightarrow 0$

$$
\begin{array}{ll}
y_{h} \rightarrow \tilde{y} & \text { in } H^{2}\left(\Omega ; \mathbb{R}^{3}\right), \\
b_{h} \rightarrow \tilde{b} & \text { in } H^{1}\left(\Omega ; \mathbb{R}^{3}\right),
\end{array}
$$

and from the weak lower semi-continuity of the energy we have

$$
\mathcal{E}_{\kappa, \theta, P}(\tilde{y}, \tilde{b}) \leq \liminf _{h \rightarrow 0} \mathcal{E}_{\kappa, \theta, P}\left(y_{h}, b_{h}\right)=\mathcal{E}_{\kappa, \theta, P}(y, b) .
$$

From the fact that a closed ball in a normed linear space is also weakly closed [48], we have $(\tilde{y}, \tilde{b}) \in \overline{\mathcal{B}}$, and the fact that there exists a unique minimizer of the energy over $\mathcal{A} \cap \overline{\mathcal{B}}$ then implies

$$
(\tilde{y}, \tilde{b})=(y, b) \text {. }
$$

Repeating next the argument of Theorem 5.1, we have from the convergence of the energy that

$$
\begin{aligned}
& y_{h} \rightarrow y \quad \text { in } H^{2}\left(\Omega ; \mathbb{R}^{3}\right), \\
& b_{h} \rightarrow b \quad \text { in } H^{1}\left(\Omega ; \mathbb{R}^{3}\right),
\end{aligned}
$$

and we can conclude that $\left(y_{h}, b_{h}\right) \rightarrow(y, b)$ for the whole sequence.

Finally, since $\left(y_{h}, b_{h}\right) \rightarrow(y, b)$ in $H^{2}\left(\Omega ; \mathbb{R}^{3}\right) \times H^{1}\left(\Omega ; \mathbb{R}^{3}\right)$ and $(y, b) \in \mathcal{B}$, we have that $\left(y_{h}, b_{h}\right) \in \mathcal{B}$ for $h$ small enough, and therefore $\left(y_{h}, b_{h}\right)$ is a local minimizer of the total energy (2.1) over $\mathcal{A}_{h}$ for all such $h$.

\section{NumericAl EXPERIMENTS}

In this section, we describe the numerical experiments we have performed. We first describe the "tent" deformation [7] and give the conditions under which this deformation is possible. Then we discuss one of its possible applications, a temperature operated valve. Our main goal is to try to qualitatively simulate the action of the valve in our computations. Finally, we discuss the issue of displaying the numerical results and present some of our graphical output.

\subsection{Conditions for a "tent"}

We assume that $e_{3}$ is a unit vector normal to the reference plane of the film. Assume that one of the sides of the film satisfies $(\nabla y \mid b) \in \mathrm{SO}(3) U_{i}$ where $U_{i}$ is one of the variants $(2.8)$. The formation of a symmetric $k$-sided tent (see Fig. 1 for a four-sided tent) is then possible [7] if and only if there exist $e \in \mathbb{R}^{3}$ such that $e \cdot e_{3}=0$ and $|e|=1$ and $Q \in \mathrm{SO}(3)$ such that

$$
\begin{gathered}
\left(Q U_{i}-I\right) e=0, \\
n \cdot U_{i}^{2} e=0, \\
\left|U_{i} n\right|>1,
\end{gathered}
$$

where $n=e \times e_{3}$, and, moreover, that $e_{3}$ is an axis of $k$-fold symmetry of the austenite. (Note that it follows from crystallography theory [3] that $k \in\{2,3,4,6\}$.) The vector $e$ is the direction of the line interface between the martensite with $(\nabla y \mid b) \in \mathrm{SO}(3) U_{i}$ and the austenite attached to the substrate. The normal to this interface is $n$. To satisfy the Hadamard condition of compatibility, we need to require (6.1). To ensure there is no shear along the interface, we need to require (6.2). Finally, (6.3) enables the film in the martensitic phase to stretch in the direction of $n$. The fact that $e_{3}$ is an axis of $k$-fold symmetry of the austenite simply allows the film to form $k$ symmetric sides of the tent. 


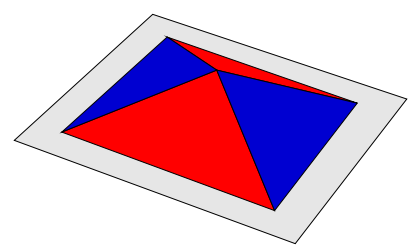

Figure 1. The "tent" deformation of the film. Outside of a square domain, the film is attached to a substrate and remains in the austenite. On the square domain, it is released and transforms to a four-sided tent. Each side of the tent satisfies $(\nabla y \mid b)=Q U_{i}$ for some $i$ where $Q \in \mathrm{SO}(3)$ and the $U_{i}$ are martensitic variants.

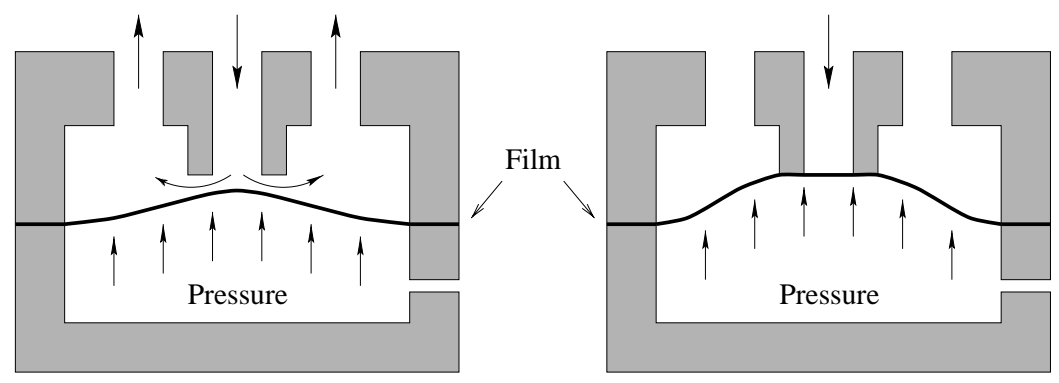

Figure 2. The temperature operated valve. At a high temperature, the film is in the austenitic phase, effectively opening the valve (left). At a low temperature, the film is in the martensitic phase, effectively closing the valve (right).

It is easily seen that the opposite sides of the symmetric four-sided tent can be chosen to belong to the same martensitic well. Hence, only two martensitic variants are needed to participate in the formation of the four-sided tent (Fig. 1).

\subsection{Application of the tent-valve}

In this section, we describe a possible application of the tent to a temperature operated valve. Consider a mechanism such as the one in Figure 2.

In a certain temperature range above the transformation temperature, the film is in the austenitic phase, effectively opening the valve. Note that the film is not completely flat due to the bias pressure in the lower part of the valve. Upon cooling the film, the pressure below the film helps it to transform into martensite and bulge up, effectively closing the valve. Heating the film again causes it to transform back to austenite and open the valve. Note that one simple way to supply heat to the film could be electric current passing through it.

\subsection{Computational example of the energy density $\phi$}

We next provide an example of an energy density satisfying all of the qualitative requirements discussed in Section 2.3. It certainly does not possess all the quantitative properties of the free energy density for a martensitic alloy, but it suffices for our computational purposes and demonstrates the expected effects.

As mentioned in Section 2.2, we will make a simple special choice of a martensitic transformation so that we do not need to rotate the reference domain $\Omega$ out of its natural position in the $x_{1} x_{2}$-plane; that is, the reference direction normal to the film is $e_{3}$, and the vectors $e$ and $n$ from (6.1)-(6.3) lie in the $x_{1} x_{2}$-plane. From the theoretical considerations in Section 6.1 (where $\kappa=0$ ), we are led to expect that only two martensitic variants appear in the deformed configuration. However, the film can transform to additional variants when the effects of surface energy are included. To confirm these expectations numerically, but to keep the model reasonably 
simple, we consider the cubic to tetragonal transformation $[4,5,35]$ given by the three transformation matrices $U_{1}, U_{2}, U_{3}$ (in the cubic basis $\left\{e_{1}, e_{2}, e_{3}\right\}$ ), where

$$
U_{1}=\left[\begin{array}{ccc}
\sqrt{1+\xi} & 0 & 0 \\
0 & 1 & 0 \\
0 & 0 & 1
\end{array}\right], \quad U_{2}=\left[\begin{array}{ccc}
1 & 0 & 0 \\
0 & \sqrt{1+\xi} & 0 \\
0 & 0 & 1
\end{array}\right], \quad U_{3}=\left[\begin{array}{ccc}
1 & 0 & 0 \\
0 & 1 & 0 \\
0 & 0 & \sqrt{1+\xi}
\end{array}\right]
$$

and where $\xi>0$.

The symmetry group $\mathcal{G}$ of the austenite is the symmetry group of the cube. It has 24 elements which are the rotations determined by rotating the orthonormal system $\left\{e_{1}, e_{2}, e_{3}\right\}$ onto another (right-handed) system $\left\{ \pm e_{\pi(1)}, \pm e_{\pi(2)}, \pm e_{\pi(3)}\right\}$ where $\pi$ is a permutation of the set $\{1,2,3\}$.

We observe that if $U=U_{1}, e=e_{2}$ and $Q$ is any rotation about $e$, then the conditions (6.1)-(6.3) are satisfied. Since $e_{3}$ is clearly a 4 -fold axis of symmetry of the cube, this configuration supports the formation of a regular 4 -sided tent with the reference configuration being a square with its sides parallel to $e_{1}$ and $e_{2}$.

It follows from (2.6) (by the polar decomposition theorem) that if $F \in \mathbb{R}_{+}^{3 \times 3}$, then $\phi(F, \theta)=\Phi(C, \theta)$ where $\Phi$ is a function of $C=F^{T} F$, the Cauchy-Green strain corresponding to the strain $F$. We will thus define below an energy density $\phi: \mathbb{R}^{3 \times 3} \times \mathbb{R} \rightarrow \mathbb{R}$ that is frame indifferent (2.6) by setting $\phi(F, \theta)=\Phi(C, \theta)$ for $\Phi: \mathbb{R}^{3 \times 3} \times \mathbb{R} \rightarrow \mathbb{R}$.

Given $\xi \geq 0$ and $F \in \mathbb{R}^{3 \times 3}$, we set $C=F^{T} F$ and define

$$
\begin{aligned}
\phi_{\xi}(F)= & \left(C_{11}+C_{22}+C_{33}-(3+\xi)\right)^{2}+\left(C_{11} C_{22} C_{33}-(1+\xi)\right)^{2} \\
& +\left(C_{11} C_{22}+C_{11} C_{33}+C_{22} C_{33}-(3+2 \xi)\right)^{2}+2 \alpha\left(C_{12}^{2}+C_{13}^{2}+C_{23}^{2}\right),
\end{aligned}
$$

where $\alpha>0$ is a constant chosen to penalize the off-diagonal elements of $C$. Note that since $\phi_{\xi}$ is a symmetric function of the diagonal elements and a symmetric function of the off-diagonal elements of $C$, it satisfies the material symmetry (2.7). Also, it is a nonnegative function of $F \in \mathbb{R}^{3 \times 3}$ and equal to zero if and only if $F \in \mathcal{U}$ where $\mathcal{U}$ is given by (2.9) and the $U_{i}$ by (6.4). Note that for $\xi=0$ the matrices (6.4) degenerate into the identity matrix $I$ and $\mathcal{U}=\mathrm{SO}(3)$.

To model the dependence on the temperature, $\theta$, we make the following simplifications. Let the temperature be allowed to range from $-\infty$ to $+\infty$ with 0 being the transformation temperature. We define the energy density $\phi$ to be the continuous function

$$
\phi(F, \theta)=\min \left\{\hat{c} \phi_{0}(F)-T(\theta), \hat{c} \phi_{\eta}(F)+T(\theta)\right\}
$$

with suitably chosen constants $\hat{c}>0$ and $\eta>0$. Here $\eta$ determines the preferred martensitic strain. To maintain the local minima for the energy density $\phi$ for all temperatures $\theta \in \mathbb{R}, T(\theta)$ is taken to be an increasing, bounded, and odd function of $\theta$. The choice of the minimum function in (6.6) does not allow $\phi$ to be differentiable everywhere, but this is more of a technical point and does not cause problems in computations. We also note that our model neglects the thermal expansion of the austenitic and martensitic strains. Finally, we remark that $\phi$ satisfies the growth condition (5.1).

\subsection{Choice of parameters}

We first give and discuss the numerical values of the parameters $\kappa, \theta, P, \eta, \alpha, \hat{c}$ and the function $T(\theta)$ that we have used for our computations. We need to mention that all of these quantities have to be chosen more or less ad hoc, since not very many experiments with various single crystal thin films have been performed yet to provide good quantitative information as to how these quantities should be chosen. Moreover, the behavior of the energy density $\phi$ away from the energy wells is far from fully understood.

We start with the strain gradient coefficient, $\kappa$. In the models of the behavior of bulk martensitic material, the size of this quantity determines the length scale of microstructure. Since the interface conditions for the 
thin film limit of the bulk model are less stringent than for the bulk model [7], we expect less microstructure in thin film configurations. Instead, possibly large deformations with a few interfaces are expected [7]. For this problem, choosing $\kappa$ on the order of $h$ results in severe penalizations of large curvatures of the deformed film which is not desirable. We set $\kappa=5 \times 10^{-5}$.

The quantity $\eta$, used in (6.6), determines the energetically preferred strains for the crystal below the transformation temperature. Our choice is $\eta=0.16$. This corresponds to, approximately, $7.7 \%$ strain, which is reasonable for martensitic alloys. Moreover, this corresponds to the height of the tent in Figure 1 being precisely 0.2 for the reference domain $\Omega=(0,1) \times(0,1)$. Of course, this height is expected to be achieved only approximately, due to the fact that the deformation cannot suffer jumps in the gradient of $y$, or in $b$ (as Fig. 1 suggests), and also due to the hydrostatic pressure under the film which causes the deformed film to take the shape of a bubble.

The values for the pressure, $P$, have been taken from the range $(0,0.2)$. We present the results for $P=0.15$.

The parameter $\alpha$ of (6.5) was set to 5. The energy itself depends little on small changes in $\alpha$ and the goal of choosing it larger than 1 was to penalize the off-diagonal terms of the Cauchy-Green strain $C$ more than its diagonal terms.

We set $\hat{c}=2 /(3 \eta)=4.1 \overline{6}$ to have the height of the barrier in the elastic energy at the transformation temperature equal to $\eta$. We remark that little is known about the barrier heights in the energy but order of $\eta$ seems reasonable.

The choice of $T(\theta)$ was

$$
T(\theta)=\frac{6}{\pi} \hat{c} \eta^{2} \arctan \theta
$$

This ensures that, at any temperature $\theta \in \mathbb{R}$, both $\mathrm{SO}(3)$ and $\mathcal{U}$ are sets of local minima of the energy density $\phi$. As $\theta \rightarrow+\infty$, the energy of the austenite lowers while the energy of the martensite grows, and the martensitic well becomes smaller. In the limit as $\theta \rightarrow+\infty$, the energy density is only minimized on the austenitic well. Similarly for $\theta \rightarrow-\infty$.

\subsection{Results and graphics}

The deformation $y \in \mathbb{R}^{3}$ provides the (immediately visualizable) deformation of the film and can be easily plotted as a two-dimensional surface. The matrix $(\nabla y \mid b) \in \mathbb{R}^{3 \times 3}$ is an argument of the free energy density $\phi$ and therefore provides information about whether the film is closer to the austenitic well or any of the martensitic ones. We follow the ideas of [21] to visualize this phenomenon. We choose grey color to correspond to the austenitic phase, red to the martensitic well corresponding to the deformation matrix $U_{1}$, blue to the martensitic well corresponding to $U_{2}$, and green to the martensitic well corresponding to $U_{3}$ (see (6.4) for the definitions of $\left.U_{i}\right)$. The darker each color, the closer is the Cauchy-Green strain $(\nabla y \mid b)^{T}(\nabla y \mid b)$ to the corresponding $U_{i}^{2}$. To determine which color to choose when coloring the surface of the film, recall the definition (6.6) of the energy density $\phi$. The choice of the minimum function there makes it easy to determine whether the deformation gradient $(\nabla y \mid b)$ resides in the austenitic well (that is, $\left.\phi(\nabla y \mid b, \theta)=\hat{c} \phi_{0}(\nabla y \mid b)-T(\theta)\right)$ or in one of the martensitic ones (that is, $\phi(\nabla y \mid b, \theta)=\hat{c} \phi_{\eta}(\nabla y \mid b)+T(\theta)$ ). In the former case, we easily determine the shade of grey by measuring the distance $\left|(\nabla y \mid b)^{T}(\nabla y \mid b)-I\right|$, while in the latter case, we first measure the distance $\left|(\nabla y \mid b)^{T}(\nabla y \mid b)-U_{i}^{2}\right|$ for each $i$ to determine the variant the Cauchy-Green strain is closest to and then use the distance $\left|(\nabla y \mid b)^{T}(\nabla y \mid b)-U_{i}^{2}\right|$ corresponding to this variant to choose the shade of red, blue or green correspondingly.

Since it is impossible to perform this at each point $x \in \Omega$, we only use certain significant points in the plotting algorithm. First, on each element we plot the resulting deformation as a piecewise linear function, using the values at the mesh points. Given that the mesh parameter $h$ is small enough, we do not lose much information this way. On each element, we choose a constant color to represent the deformation gradient by evaluating the matrix $(\nabla y \mid b)$ at the quadrature points used in the minimization algorithm and forming the weighted average 


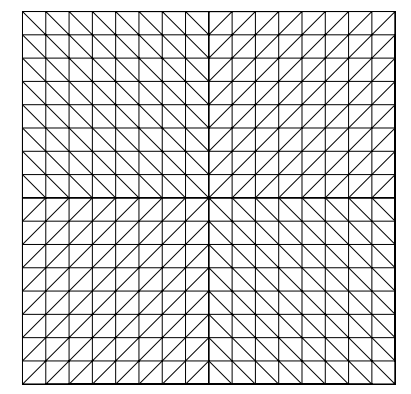

Figure 3 . The finite element triangular mesh used for modeling the valve.

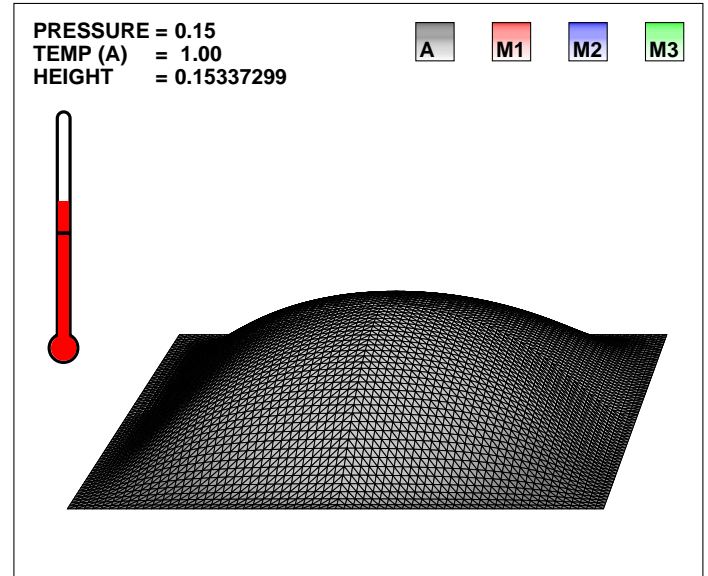

(a)

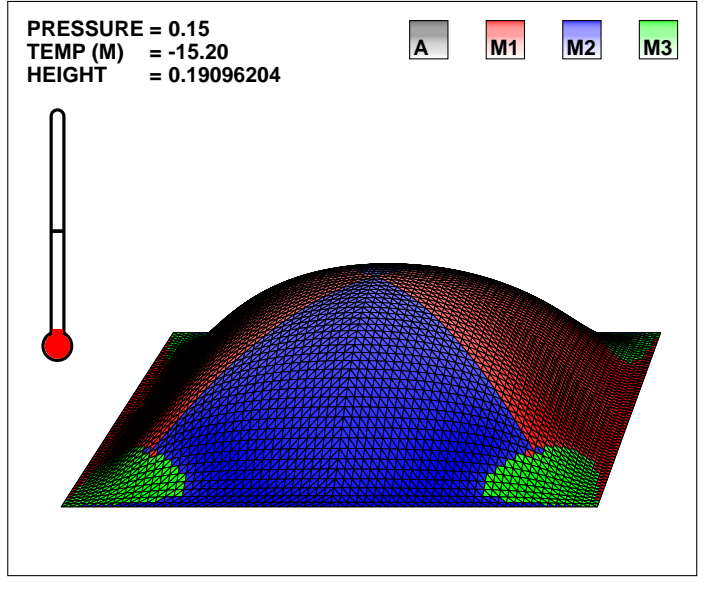

(b)

Figure 4 . The film at a high temperature austenitic phase (a) and a low temperature martensitic phase (b). At a high temperature, the film is in the austenite (a). Upon cooling, it transforms to the martensite and pops up (b). The grey color corresponds to the austenite (the deformation gradient $(\nabla y \mid b)$ is in the austenitic energy well), while the red, blue and green colors correspond to the individual variants of martensite (the transformation matrices $U_{1}, U_{2}$ and $U_{3}$ of (6.4), respectively). The results shown were obtained using the reduced HCT triangle.

of the obtained values with the weights from the quadrature rule. Again, as $h$ becomes small, this gives a reasonably good approximation.

We performed temperature and pressure continuations, thus obtaining sequences of images. For better visualization purposes we created animated files in the GIF and MPEG formats. These are publicly available at the website [10].

We now give a complete description of an individual frame of these animated files (see Fig. 4, for an example of these frames). The reference configuration is taken to be $(0,1) \times(0,1)$. We use a mesh (Fig. 3) that fits the symmetry of the "tent" configuration [7]. In the upper left corner, we display the following three parameters: pressure $P$, temperature $\theta$, and the height of the deformed film at the center of $\Omega$, that is, the value $y_{3}(0.5,0.5)$. The letter $\mathrm{A}$ in the parentheses after the word TEMP indicates that the temperature is above the transformation temperature and austenite is the global minimizer of the free energy density $\phi$. Similarly, $\mathrm{M}$ indicates the temperature is below the transformation temperature and martensite is the global minimizer 
of $\phi$. Since the film resides in a local minimum of the total energy (2.1) for a certain temperature range in our model and the deformation does not change with the temperature, we include a schematic thermometer on the left-hand side of the frame, displaying the temperature. In the animated files, this shows the change in the temperature while the deformation stays constant. The transformation temperature is indicated by the mark on the thermometer.

In the upper right corner of the frame, we schematically show the colors corresponding to each variant. Here, A stands for the austenite and $\mathrm{M} i$ for the $i$ th variant of the martensite. Finally, note that each frame is displayed with the corresponding finite element mesh.

The Morley triangle is the simplest triangular element one could use to solve fourth order problems. It is known that the smallest polynomial space that needs to be included in the function space $\mathcal{P}$ for the method to converge is $\mathcal{P}_{2}$ [17], which is exactly the space of functions of the Morley element. However, the functions in the Morley finite element space are not continuous across the inter-element edges, hence the Dirichlet boundary conditions for $y$ are not imposed everywhere along the boundary. This means that at the corners of the square domain that we use in the numerical simulations, the imposed boundary conditions do not force the deformation gradient $(\nabla y \mid b)$ to be the identity, which is a required compatibility condition (recall that outside the square the film is attached to a substrate and hence remains in the austenite). Since the corners should then, quite reasonably, provide a mechanism for the film to transform back to austenite upon heating, we do not feel that the Morley element is a good choice for our problem. In fact, numerical simulations using this element showed great instability of the temperature for which the film changes phase.

Hence, in the remainder of this paper, we give results corresponding to the Zienkiewicz triangle and the reduced Hsieh-Clough-Tocher triangle. Note that these two elements have exactly the same degrees of freedom. That means that with no additional work at all, the results from the (conceivably faster) Zienkiewicz method can be used as an initial guess (or preconditioner) for the (conceivably slower) HCT method.

The numerical experiment itself proceeds as follows. We fix the pressure $P=0$, a temperature $\theta$ above the transformation temperature, and take as the initial guess

$$
\left.\begin{array}{rl}
\left(y_{1}\left(x_{1}, x_{2}\right), y_{2}\left(x_{1}, x_{2}\right), y_{3}\left(x_{1}, x_{2}\right)\right) & =\left(x_{1}, x_{2}, 0\right) \\
\left(b_{1}\left(x_{1}, x_{2}\right), b_{2}\left(x_{1}, x_{2}\right), b_{3}\left(x_{1}, x_{2}\right)\right) & =(0,0,1)
\end{array}\right\} \text { for }\left(x_{1}, x_{2}\right) \in \Omega .
$$

This is clearly a (unique) global minimizer of the total energy when $P=0$. We then use a zero-order continuation in the pressure to obtain an energy minimizer for $P>0$, that is, we increase the pressure in small steps, taking the minimizing deformation for the previous smaller value of the pressure as the initial guess for the current value of the pressure, and apply the minimization technique described in Section 4. When the desired value for the pressure is reached, we fix the pressure and perform the same kind of continuation on the temperature. We note that the final pressure $(P=0.15)$ has been chosen large enough so that the film transforms to martensite easily as the temperature is increased, but is small enough so that the film remains in austenite at lower temperatures.

First, the temperature is lowered below the transformation temperature until the film fully transforms into martensite and pops up, and then the temperature is increased above the transformation temperature until the film transforms into austenite. The two resulting states for the reduced HCT triangle are shown in Figure 4. We do not show the deformation for the Zienkiewicz element since the differences between the obtained deformations are almost indiscernible. Starting at a temperature above the transformation temperature, the film is in the austenite (Fig. 4(a)). In a certain temperature range below this temperature, the deformation of the film remains constant. This is because in the energy density function (6.6) only the function $\hat{c} \phi_{0}(F)-T(\theta)$ is active. When the temperature is lowered enough so that the function $\hat{c} \phi_{\eta}(F)+T(\theta)$ becomes active, the film transforms into martensite and pops up (Fig. 4(b)). Similarly, if, after this point, the temperature is increased, the film remains in the martensite until the temperature reaches a critical temperature above the transformation temperature and the film transforms back to austenite and the deformation comes back to the one with which the temperature continuation was started. This leads to a nearly rectangular hysteresis loop in the height of the deformed film. 


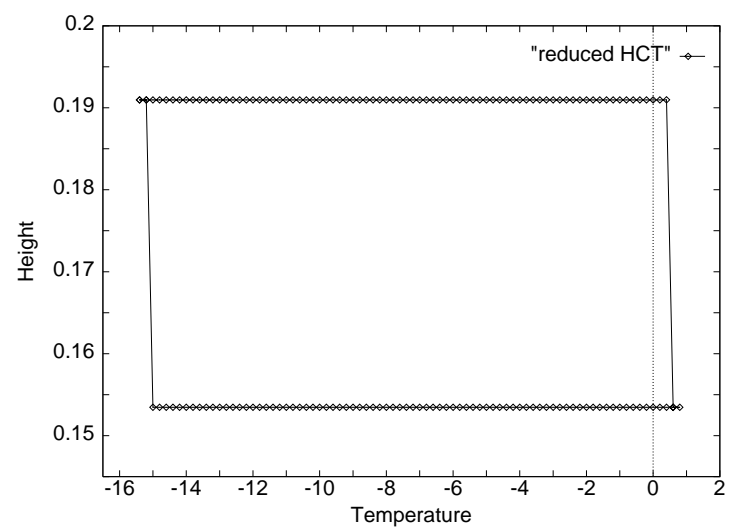

Figure 5. Hysteresis in the height of the deformed film as the temperature changes. The results shown are for the reduced HCT triangle on a $64 \times 64$ mesh, for pressure $P=0.15$, and the temperature continuation step 0.2 .

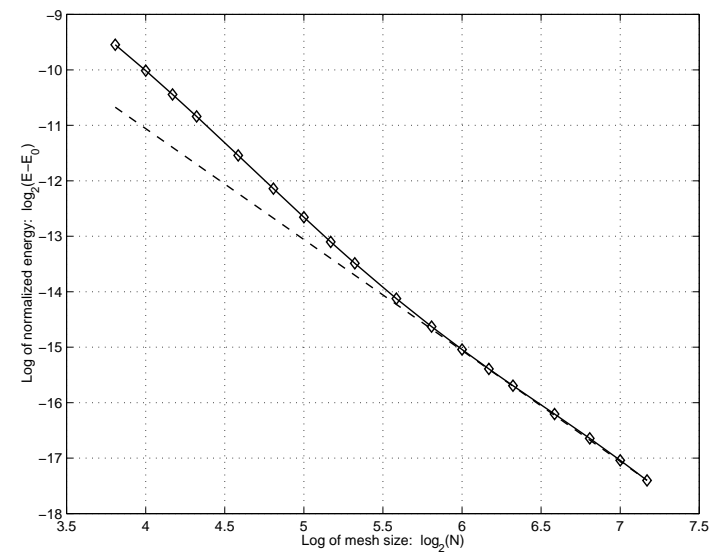

Figure 6. The computed energy of the film for the reduced HCT triangle in the austenite (at the transformation temperature $\theta=0$ ) plotted as a function of the grid size. The approximation of the computed energy by the dashed line given by $-6.758 \times 10^{-3}+1.2 \times 10^{-1} N^{-2}$ where $h=1 / N$ shows the quadratic convergence of Theorem 5.3.

For all of the finite elements used and for all mesh sizes, we have obtained the nearly rectangular hysteresis loop such as the one shown in Figure 5 for the reduced HCT triangle with mesh $64 \times 64$ and pressure $P=0.15$. That is, the height of the film changes abruptly during one temperature step. Thus, we see that for each temperature included in the hysteresis loop we obtain two isolated local minima for the energy.

While the energy, the heights of the film in the austenite and the martensite, and the temperature at which the film transforms from the martensite to the austenite converge or remain stable as the mesh gets refined, the temperature at which the film transforms from the austenite to the martensite seems sensitive to the mesh size and the finite element (and possibly the quadrature rule) used. We demonstrate these phenomena in Figures 6-9.

For the micro-valve, it would be desirable to make the width of the hysteresis loop as small as possible, that is, to make the two temperatures for which the film changes phase as close to each other as possible. This might be accomplished by choosing a larger value for the pressure or by choosing a softer alloy (one with smaller elastic moduli) or by having shallower energetic wells. 


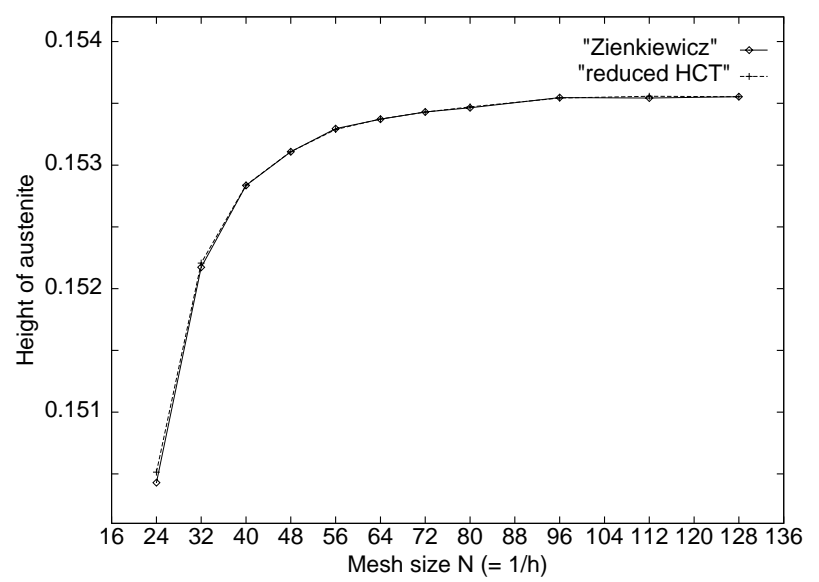

Figure 7 . The height of the film in the austenite plotted as a function of the grid size. The results shown are for both the Zienkiewicz triangle and the reduced HCT triangle.

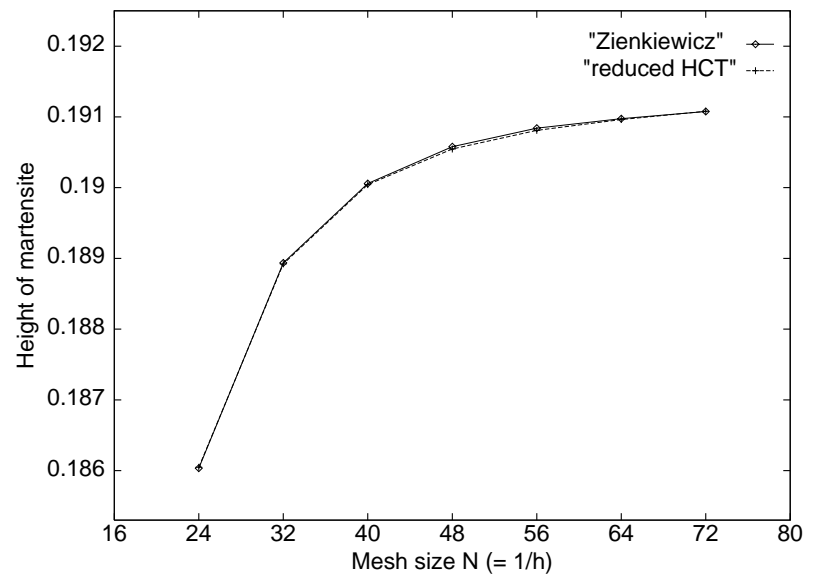

Figure 8. The height of the film in the martensite plotted as a function of the grid size. The results shown are for both the Zienkiewicz triangle and the reduced HCT triangle.

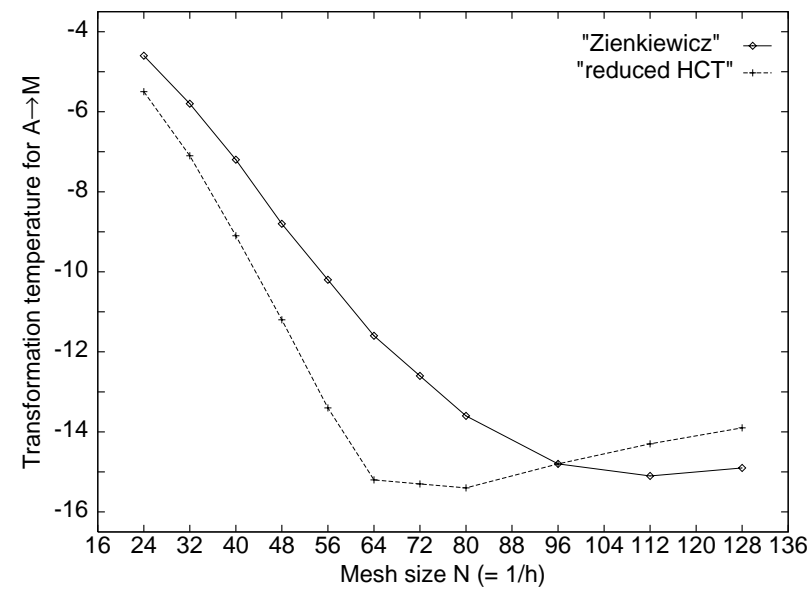

Figure 9. The critical temperature at which the transformation from the austenite to the martensite occurs (and the film pops up) as a function of the grid size. The results shown are for both the Zienkiewicz triangle and the reduced HCT triangle. 


\section{REFERENCES}

[1] R. Adams, Sobolev spaces. Academic Press, New York (1975).

[2] J.H. Argyris, I. Fried and D.W. Scharpf, The TUBA family of plate elements for the matrix displacement method. Aero. J. Roy. Aero. Soc. 72 (1968) 701-709.

[3] N.W. Ashcroft and N.D. Mermin, Solid State Physics. Saunders College Publishing, Orlando (1976).

[4] J. Ball and R. James, Fine phase mixtures as minimizers of energy. Arch. Rat. Mech. Anal. 100 (1987) 13-52.

[5] J. Ball and R. James, Proposed experimental tests of a theory of fine microstructure and the two-well problem. Phil. Trans. R. Soc. Lond. A 338 (1992) 389-450.

[6] M. Bernadou and K. Hassan, Basis functions for general Hsieh-Clough-Tocher triangles, complete or reduced. Internat. J. Numer. Methods Engrg. 17 (1981) 784-789.

[7] K. Bhattacharya and R.D. James, A theory of thin films of martensitic materials with applications to microactuators. J. Mech. Phys. Solids 47 (1999) 531-576.

[8] K. Bhattacharya, B. Li and M. Luskin, The simply laminated microstructure in martensitic crystals that undergo a cubic to orthorhombic phase transformation. Arch. Rat. Mech. Anal. 149 (1999) 123-154.

[9] S.C. Brenner and L.R. Scott, The mathematical theory of finite element methods. Springer-Verlag, New York (1994).

[10] P. Bělík, T. Brule and M. Luskin, Numerical modelling of a temperature-operated martensitic microvalve. http://www.math. umn.edu/ luskin/research/valve/.

[11] P. Bělík and M. Luskin, Stability of microstructure for tetragonal to monoclinic martensitic transformations. ESAIM: M2AN 34 (2000) 663-685.

[12] C. Carstensen and P. Plecháč, Numerical solution of the scalar double-well problem allowing microstructure. Math. Comp. 66 (1997) 997-1026.

[13] C. Carstensen and P. Plecháč, Adaptive algorithms for scalar non-convex variational problems. Appl. Numer. Math. 26 (1998) 203-216.

[14] M. Chipot, C. Collins and D. Kinderlehrer, Numerical analysis of oscillations in multiple well problems. Numer. Math. 70 (1995) 259-282.

[15] M. Chipot and D. Kinderlehrer, Equilibrium configurations of crystals. Arch. Rat. Mech. Anal. 103 (1988) $237-277$.

[16] M. Chipot and S. Müller, Sharp energy estimates for finite element approximations of nonconvex problems. Preprint (1997).

[17] P.G. Ciarlet, The finite element method for elliptic problems. North-Holland, Amsterdam (1978).

[18] R.W. Clough and J.L. Tocher, Finite element stiffness matrices for analysis of plates in bending. In Proceedings of the conference on matrix methods in structural mechanics. Wright Patterson A.F.B., Ohio (1965) 515-545.

[19] C. Collins, Computation of twinning. In Microstructure and phase transitions. J. Ericksen, R. James, D. Kinderlehrer and M. Luskin Eds. IMA Vol. Math. Applic. 54, Springer-Verlag, New York (1993) 39-50.

[20] C. Collins and M. Luskin, The computation of the austenitic-martensitic phase transition. In Partial differential equations and continuum models of phase transitions. M. Rascle, D. Serre and M. Slemrod Eds. Lect. Notes Phys. 344, Springer-Verlag, Berlin (1989) 34-50.

[21] C. Collins, M. Luskin and J. Riordan, Computational results for a two-dimensional model of crystalline microstructure. In Microstructure and phase transitions. J. Ericksen, R. James, D. Kinderlehrer and M. Luskin Eds. IMA Vol. Math. Applic. 54, Springer-Verlag, New York (1993) 51-56.

[22] G. Dolzmann, Numerical computation of rank-one convex envelopes. SIAM J. Numer. Anal. 36 (1999) 1621-1635.

[23] J.W. Dong, L.C. Chen, C.J. Palmstrøm, R.D. James and S. McKernann, Molecular beam epitaxy growth of ferromagnetic single crystal (001) $\mathrm{Ni}_{2} \mathrm{MnGa}$ on (001) GaAs. Appl. Phys. Lett. 75 (1999) 1443-45.

[24] D.A. Dunavant, k High degree efficient symmetrical Gaussian quadrature rules for the triangle. Internat. J. Numer. Methods Engrg. 21 (1985) 1129-1148.

[25] G.B. Folland, Real analysis. Modern techniques and their applications. John Wiley \& Sons, Inc., New York (1984).

[26] M. Giaquinta. Calculus of variations. Springer-Verlag, Berlin (1996).

[27] D. Gilbarg and N. Trudinger, Elliptic partial differential equations of second order. Springer-Verlag, Berlin (1998).

[28] R. Glowinski, Numerical methods for nonlinear variational problems. Springer-Verlag, New York (1984).

[29] P.-A. Gremaud, Numerical analysis of a nonconvex variational problem related to solid-solid phase transitions. SIAM J. Numer. Anal. 31 (1994) 111-127.

[30] M. Gurtin, Topics in finite elasticity. SIAM, Philadelphia (1981).

[31] R.D. James and R. Rizzoni, Pressurized shape memory thin films. J. Elasticity 59, special issue in honor of Roger Fosdick, D. Carlson Ed. (2000) 399-436.

[32] P. Krulevitch, A.P. Lee, P.B. Ramsey, J.C. Trevino, J. Hamilton and M.A. Northrup, Thin film shape memory alloy microactuators. Journal of Microelectromechanical Systems 5 (1996) 270.

[33] M. Kružík, Numerical approach to double well problems. SIAM J. Numer. Anal. 35 (1998) 1833-1849.

[34] P. Lascaux and P. Lesaint, Some nonconforming finite elements for the plate bending problem. Rev. Française Automat. Informat. Recherche Opérationnelle Sér. Rouge Anal. Numér. R-1 (1975) 9-53. 
[35] B. Li and M. Luskin, Finite element analysis of microstructure for the cubic to tetragonal transformation. SIAM J. Numer. Anal. 35 (1998) 376-392.

[36] B. Li and M. Luskin, Nonconforming finite element approximation of crystalline microstructure. Math. Comp. 67 (1998) 917-946.

[37] B. Li and M. Luskin, Approximation of a martensitic laminate with varying volume fractions. ESAIM: M2AN 33 (1999) 67-87.

[38] Z. Li, Simultaneous numerical approximation of microstructures and relaxed minimizers. Numer. Math. 78 (1997) 21-38.

[39] D.G. Luenberger, Introduction to linear and nonlinear programming. Addison-Wesley, Reading, Mass. (1973).

[40] M. Luskin, Approximation of a laminated microstructure for a rotationally invariant, double well energy density. Numer. Math. 75 (1996) 205-221.

[41] M. Luskin, On the computation of crystalline microstructure. Acta Numer. 5 (1996) 191-257.

[42] M. Luskin and L. Ma, Analysis of the finite element approximation of microstructure in micromagnetics. SIAM J. Numer. Anal. 29 (1992) 320-331.

[43] L.S.D. Morley, The triangular equilibrium element in the solution of plate bending problems. Aero. Quart. 19 (1968) $149-169$.

[44] P. Pedregal, On the numerical analysis of non-convex variational problems. Numer. Math. 74 (1996) 325-336.

[45] E. Polak, Computational methods in optimization. Academic Press, New York (1971).

[46] T. Roubíček, Numerical approximation of relaxed variational problems. J. Convex Anal. 3 (1996) 329-347.

[47] H.L. Royden, Real analysis. 3rd edn, Macmillan Publishing Company, New York (1988).

[48] W. Rudin, Functional analysis. McGraw-Hill, New York (1973).

[49] Z. Shi, Error estimates of Morley element. Chinese J. Num. Math. Appl. 12 (1990) 102-108.

To access this journal online: www.edpsciences.org 